\title{
Reversals of coherent structures in turbulent mixed convection
}

\author{
Michael Mommert ${ }^{1,2,} \dagger$, Daniel Schiepel $^{1}$, Daniel Schmeling ${ }^{1}$ \\ and Claus Wagner ${ }^{1,2}$ \\ ${ }^{1}$ Institute of Aerodynamics and Flow Technology, German Aerospace Center (DLR), \\ Bunsenstr. 10, 37073 Göttingen, Germany \\ ${ }^{2}$ Institute of Thermodynamics and Fluid Mechanics, Technische Universität Ilmenau, \\ PF 100565, 98684 Ilmenau, Germany
}

(Received 17 February 2020; revised 10 August 2020; accepted 19 August 2020)

Reconfiguration events in turbulent mixed convection, i.e. the superposition of thermal and forced flow contributions, at the two different Richardson numbers $R i=1.5$ and $R i=3.7$ and similar Rayleigh numbers of $R a \approx 10^{8}$ are investigated with tomographic particle image velocimetry in combination with local temperature measurements. For both cases, the three-dimensional velocity fields reflect diagonally aligned large-scale circulations (LSC) switching their alignment by rotating their axes around a pivot located at the centre of the LSC, while the temperatures perform a translation movement of the structures in agreement with earlier temperature-based investigations. For the high $R i$ case, the switching process of the observed spontaneous reconfigurations is induced by a reversing thermal flow contribution while the forced flow contribution is constant. Furthermore, it is shown that a secondary roll structure, which drives the reconfiguration process in Rayleigh-Bénard convection, also exists in mixed convection. However, in the latter, the flow reversals are triggered by different structures which accumulate and release their kinetic energy according to a proper orthogonal decomposition analysis. In contrast, for the low $R i$ case, the structure formation during continuous reconfigurations is governed by a Taylor- or Görtler-type instability. This means that the forced convection substantially affects the reconfiguration mechanism of these structures. Therefore, the reconfigurations cannot be described by a simple superposition of structures associated with the two flow contributions as for the high $R i$.

Key words: buoyancy-driven instability, convection in cavities, turbulent convection

\section{Introduction}

\subsection{Flow reconfigurations in thermal convection}

Thermal convection affects a wide spectrum of flows reaching from geophysical to engineering matters. Typically, these flows are characterised by large-scale circulations

$†$ Email address for correspondence: michael.mommert@ dlr.de 
(LSC) and their dynamical behaviour represents a current research topic. Our aim is to show to which extent model concepts for pure thermal convection apply to cases of mixed convection where thermal convection is superimposed by forced convection. Such model concepts exist for Rayleigh-Bénard convection (RBC) for a variety of regimes and geometries:

Villermaux (1995) developed a model explaining oscillating instabilities in two-dimensional square samples as a coupling between the bottom and top boundary layers: as plumes emitted from one boundary layer impinge on the other boundary layer, they cause an instability, leading to a new emission of a plume with the opposite temperature deviation and flow direction. In cases of resonance, this process manifests itself in the form of temperature oscillations. Experimental studies in three-dimensional cylindrical samples agree with the model concept of Villermaux. These studies comprise in particular time correlations of local temperature and velocity measurements (Qiu et al. 2004) as well as cross-sectional velocity fields (Sun, Xia \& Tong 2005).

So far, this model only determines an intermittent heat transport as a cause of temperature oscillations, whereas the direction of the mean wind is considered to be consistent. In another approach, torsional (Funfschilling \& Ahlers 2004; Funfschilling, Brown \& Ahlers 2008) and sloshing (Xi et al. 2009) modes of the LSC are identified as cause of the temperature oscillations in cylindrical samples. By combining both modes, temperature and velocity oscillations can be described as waves of the LSC's location. This concept replaces the model of a resonating plume emission (Brown \& Ahlers 2009; Xi et al. 2009).

Besides oscillations, reversals present another type of flow instabilities. They fundamentally affect the orientation of the LSC. Further, they occur non-periodically and on longer time scales than the oscillations (Cioni, Ciliberto \& Sommeria 1997; Niemela et al. 2001; Sreenivasan, Bershadskii \& Niemela 2002). All three referenced studies were carried out in cylindrical samples equipped with a sensor array to provide local temperature information which also allows one to draw conclusions on the global velocity field. As this geometry prefers no particular LSC orientation, the following is assumed regarding the cause of these events: when one large plume or several smaller plumes eject a large amount of heat from the boundary layer, its complete buoyancy potential can be consumed. Subsequently, the direction of an impinging fluid package can redetermine the direction in which the boundary layer ejects new plumes (Niemela et al. 2001).

In addition to the previous local considerations, the LSC dynamics can also be described globally. Sreenivasan et al. (2002) present the concept of a flow in a cylindrical sample with two stable states distinguished by different rotation directions of the LSC. Its instability is caused by an imbalance between buoyancy and friction. In other words, reorientations of the LSC result from turbulent fluctuations overcoming a flow-stabilising potential barrier.

Yet, this concept is based on the assumption that two discrete states exist, while cylindrical samples allow for continuous changes of the LSC orientation. Brown \& Ahlers (2006) consider this option to classify the LSC reconfiguration events in rotations and cessations. Their statistical investigations reveal that both types of events occur in a Poisson-distributed manner. Consequently, the events are considered as spontaneous and independent from earlier occurrences. Despite the similar statistics of both types, their mechanisms are reported to be different: rotations describe events, in which the LSC conserves its momentum but the rotation axis reorients in a continuous process. In contrast, the LSC breaks down completely and re-emerges in a direction independent from its previous direction during cessations. The breakdown of the LSC during a cessation event is confirmed by Xi \& Xia (2007). They show the absence of the LSC based on 
the decoherence of a 2-D velocity field measured by particle image velocimetry (PIV). Further, Xie, Wei \& Xia (2013) show that both rotations and cessations also occur in fluids with high Prandtl numbers, but the associated azimuthal velocity of the LSC is orders of magnitude lower than in the studies with lower Prandtl numbers.

Although the distinction between events like cessations or reversals, which heavily affect the LSC, and oscillations, which only have a weak effect on the LSC orientation, seems strict, some flows exhibit instabilities which blur this classification: for instance, Resagk et al. (2006) and Brown \& Ahlers (2009) show measurements of LSC oscillations with substantial azimuthal amplitudes of up to $120^{\circ}$, which highlight the potential influence of oscillation-type instabilities.

More recent studies reduced the degrees of freedom for the LSC's orientation by using a rectangular (quasi) two-dimensional sample (Sugiyama et al. 2010; Podvin \& Sergent 2015; Castillo-Castellanos, Sergent \& Rossi 2016; Podvin \& Sergent 2017; Castillo-Castellanos et al. 2019; Chen et al. 2019). These studies include both numerical and experimental investigations aimed at gaining an insight into the mechanisms of the reversal events. The results of this sample type highlight the role of secondary corner circulations: they drive the reversal process as they grow in size and in terms of kinetic energy. This process continues until the secondary corner circulations are big enough to cut off the diagonal LSC and form a new LSC rotating in the opposite direction (Sugiyama et al. 2010). Further understanding of this process was generated by means of proper orthogonal decompositions (POD) (Podvin \& Sergent 2015, 2017) and a global energy and momentum development analysis (Castillo-Castellanos et al. 2016). Their main findings include the detection of a precursor mode connecting the onset of a reversal to a sign change in the time development coefficient of the mode which connects the boundary layer with the bulk flow. Castillo-Castellanos et al. (2019) confirm that the reversals occur as a part of a successive process which may, however, take different paths within the POD phase space. Experimental investigations by Chen et al. (2019) further point out that the fluctuation strength of the LSC itself is the main determining factor of the reconfiguration rate, while the corner circulations are still a symptom of the process.

The studies of Huang et al. (2015) and Zhang et al. (2020) reveal how sensitively these (quasi) two-dimensional flows react to changes in the boundary conditions. Huang et al. (2015) compare RBC in samples with a constant temperature and heat flux at the bottom plate, while maintaining a constant temperature at the top plate. Contrary to their expectations, more reversals occurred for the constant temperature boundary condition at both plates than for the set-up with the constant heat flux boundary condition at the bottom plate. This case also exhibited a stronger LSC and weaker temperature fluctuations, which is why the authors postulate that the reversals are driven by a force which restores the broken symmetry of an unidirectional LSC over the course of time. Moreover, Zhang et al. (2020) examined the flow in a sample featuring control regions with a constant temperature on otherwise adiabatic sidewalls and demonstrate that their position allows one to either enhance or suppress the occurrence of reversals. The latter can be explained by a weakening of plumes as the control regions remove additional heat from the plumes or cause a separation from the sidewall.

To specifically exclude the influence of the secondary corner circulations on flow reversals, a thin cylindrical sample with a horizontal centre axis was investigated by Wang et al. (2018). Even without the corner circulations, flow reversals were observed. In this case, a heat accumulation followed by a massive plume emission interrupts the LSC's stable flow structure. Chen, Wang \& Xi (2020) also pursue the idea of eliminating the influence of corner vortices. By adding chamfer inserts to a quasi-two-dimensional sample, 
they describe a reversal type induced by the instability of the main vortex that also occurs in unmodified samples but with a lower frequency.

Considering a range of small aspect ratios of rectangular samples, Huang \& Xia (2016) show the reversal behaviour in the transition from quasi-two-dimensional to three-dimensional flow. They find that reversals occur more frequently in samples with smaller aspect ratios, in which the plumes are forced to travel through the bulk region and thus disturb the LSC more often due to the geometrical confinement.

When it comes to three-dimensional cubic samples, investigations of the dynamics of the LSC were conducted numerically (Foroozani et al. 2017) and experimentally (Bai, Ji \& Brown 2016). Both studies reveal that the LSC changes its alignment along the diagonals of the sample. This process is characterised by the rotation of the LSC's orientation during a short transient period. While rotations of $180^{\circ}$ occurred, no cessations including a breakdown of the LSC were detected. Vasiliev et al. (2019) discuss the role of actual azimuthal flow during these events. They find that events with significant azimuthal angular moment exist but they are not necessarily associated with the reorientations of the LSC. Hence, they propose a model based on the superposition of two perpendicular angular momenta parallel to the sample walls. The model allows one to describe the process as a reversal of one of these angular momentum components and without azimuthal flow components. The findings of Soucasse et al. (2019) are in agreement with this idea as the there applied POD yielded modes representing the proposed superimposing circulations. Further, the dynamics of higher modes again suggests a destabilising behaviour of the corner circulations.

This overview on the different LSC reconfiguration processes reveals that their driving mechanism can be different for different boundary conditions. Another example for this is the frequency of the occurrence of reconfigurations, which shows different dependencies on the Rayleigh number in the above-mentioned studies: increasing Rayleigh numbers are associated with increasing (Araujo, Grossmann \& Lohse 2005), decreasing (Ni, Huang \& Xia 2015; Wang et al. 2018; Chen et al. 2019, 2020), non-monotonic (Brown \& Ahlers 2006) or independent (Xi \& Xia 2007) behaviour of the occurrence frequencies.

\subsection{Mixed convection flows in rectangular samples}

To study the effects of the superposition of thermal and forced convection, we conducted experiments in an RBC-like rectangular sample with added in- and outlet vents, see § 2.1. This corresponds to the trend of investigating flows with closer connections to engineering problems (Xia 2013). As displayed in figure 1(c), Kühn et al. (2012) report that this flow arranges itself in a zigzag-like structure. This means the single longitudinal convection roll (figure 1b), which exists in pure forced convection (Schmeling et al. 2011), realigns in the shape of multiple diagonal segments or LSCs caused by the additional buoyancy forces. Furthermore, these LSCs can be observed as counter-rotating circulations in a vertical longitudinal section (Westhoff et al. 2010). They then have a similar appearance as the multiple LSCs of pure RBC in the same geometry, see figure 1(a) (Kaczorowski \& Wagner 2009; Podvin \& Sergent 2012).

An analogy to this break up of a longitudinal roll can be found in turbulent mixed convection channel flow (Pirozzoli et al. 2017; Blass et al. 2020). There, the heat transporting convection rolls align longitudinally to the main flow direction but start to meander at $R i \approx 10^{\circ}$. This observed instability is similar to the wavy instability, which Clever \& Busse (1991) explain and Pabiou, Mergui \& Bénard (2005) prove for laminar flows by means of experiments. 
(a)

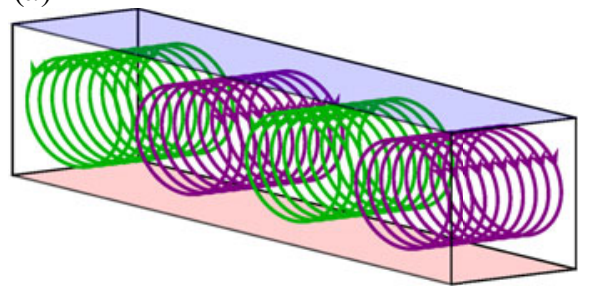

(b)

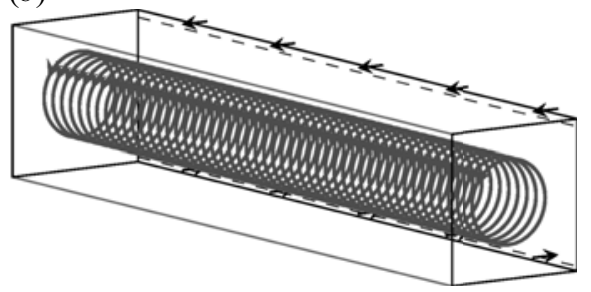

(c)

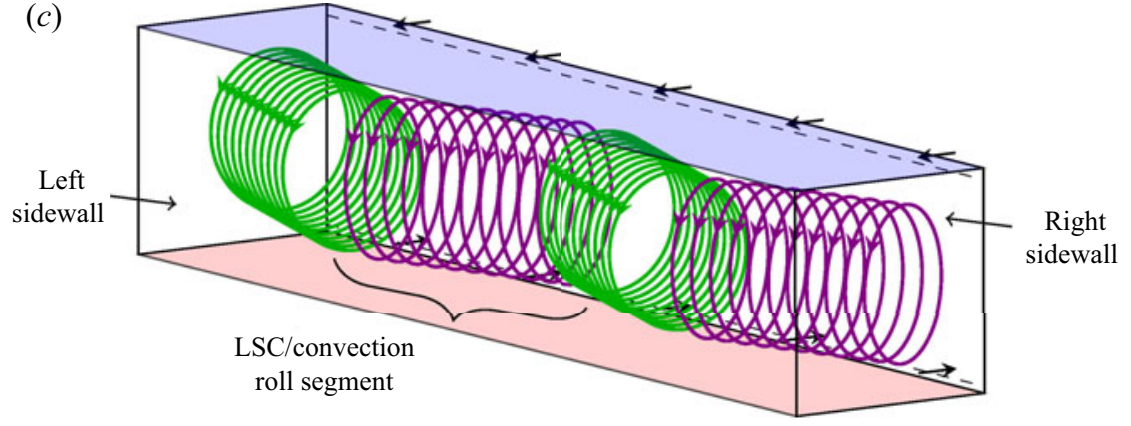

FIGURE 1. Comparison of the conceptual flow structures of pure RBC (a), pure forced convection $(b)$ and mixed convection $(c)$ in a rectangular sample. The flow structures are visualised by generic streamlines (green, purple, black). The respective forcing is indicated by the heated (red) and cooled (blue) faces $(a, c)$ as well as by flow arrows at the in- and outlet at the rear wall $(b, c)$. For mixed convection $(c)$, the flow structure aligns in the shape of a zigzag with multiple convection roll segments or LSCs according to Kühn et al. (2012). The displayed formation comprises four LSCs spanning between the sidewalls.

However, regarding turbulent mixed convection in the here considered cuboidal convection sample, Westhoff et al. (2010) measured a low frequency oscillation of the fluid temperature at the outlet of the sample and also show that the roll core positions in different cross-sections vary over time. In the following, Schmeling, Bosbach \& Wagner (2013) identify different types of flow instabilities by means of temperature measurements using a sensor array positioned in the sample. The resulting temperature fields exhibit hot and cold spots representing sections of the up- and down-welling flow of the zigzag roll structure. Based on the temperature time series of single probes, two types of dynamical behaviour are distinguished: continuous temperature oscillations $(\mathcal{C})$ and spontaneous $(\mathcal{S})$ events occurring stochastically on longer time scales. For both types, the temperature signals indicate that the hot and cold spots move through the sample along the longitudinal direction. Projecting these motions on the zigzag roll structure implies a travelling of the roll segments. Accordingly, roll segments emerge and break down at the opposing sidewalls in this model conception. A specific event of type $\mathcal{S}$ was observed by Westhoff (2012, pp. 54-70) performing long-time two-dimensional, two-component PIV measurements in the central longitudinal section of the sample. The subsequent POD shows changes between four-roll and three-roll states, respectively, associated with the first and second modes of the decomposition. These findings are in agreement with the proposed process of Schmeling et al. (2013).

Since the causes of both dynamical behaviours have not yet been identified in existing research, the understanding of these processes will also benefit from the intended transfer of RBC model concepts. Consequently, we conducted tomographic PIV measurements 
with simultaneous temperature measurements of both $\mathcal{C}$ - and $\mathcal{S}$-type events to contribute to the understanding of the process. The results were examined with respect to parallels to instabilities found in RBC. In particular, PODs were conducted on the basis of the velocity fields obtained during the reconfiguration events. This analysis allows us to discuss the influence of the resulting coherent structures in the style of the approach of Podvin \& Sergent (2015).

\section{Experimental set-up}

\subsection{Mixed convection sample}

We investigated the flow in an enclosure with the dimensions $L=2500 \mathrm{~mm}$ and $H=$ $W=500 \mathrm{~mm}$ defining the aspect ratios $\Gamma_{X Y}=5$ and $\Gamma_{Y Z}=1$. A sketch of the sample is presented in figure 2 which also comprises the experimental instrumentation, see $\S 2.2$. The temperature boundary conditions of the sample were defined by a blackened aluminium bottom plate $\left(T_{H P}\right)$ heated by tempered water and a top plate $\left(T_{C P}\right)$ of the same material passively cooled to room temperature. The lateral faces were double-walled by $10 \mathrm{~mm}$ thick polycarbonate to minimise heat exchange with the surroundings while allowing optical access. A $25 \mathrm{~mm}$ high inlet vent (A) was positioned along the top edge of the rear wall, and a $15 \mathrm{~mm}$ high outlet (B) at the bottom edge. This set-up allowed us to study mixed convection, as both buoyancy and inertial forces can be induced in the fluid sample. The direction of the forced flow is indicated by the arrows inside the ducts in figure 2 .

The prevalent flow state in the sample depends on the dimensionless parameters representing the thermal forcing $R a=g \beta \Delta T H^{3} /(v \alpha)$, the inertial forcing $R e=v_{\text {in }} H / \nu$ and the relation between momentum and heat transport, namely the Prandtl number $P r=$ $\nu / \alpha$. With air as working fluid, $\operatorname{Pr} \approx 0.7$ was assumed to stay constant for the investigated parameter range. The other dimensionless parameters were varied by changing the temperature difference between the top and bottom plates $\Delta T=T_{H P}-T_{C P}$ and the mean inflow velocity $v_{i n}$. In particular, we adjusted $\Delta T$ through $T_{H P}$ as the top plate was passively cooled. Moreover, the volume flow rate $\dot{V}$ of the air entering the sample via the opening of the inlet $A_{\text {in }}$ determines the mean inflow velocity $v_{\text {in }}=\dot{V} / A_{\text {in }}$. Except for the sample height $H$ and gravitational acceleration $g$, all other quantities determining the dimensionless numbers were material parameters: they comprise the thermal expansion coefficient $\beta$, the kinematic viscosity $v$ and the thermal diffusivity $\alpha$.

In order to generate a sufficiently developed velocity profile at the inlet, the inlet channel had a length equalling 30 times its height. The first $100 \mathrm{~mm}$ were equipped with aluminium honeycomb material with an inner diameter of $3 \mathrm{~mm}$ in order to homogenise the flow. Further details of the sample were described by Kühn et al. (2011) regarding the realisation of the apparatus, and by Schmeling et al. (2013) regarding the characterisation of the experiment's boundary conditions.

Schmeling et al. (2013) also presented a set of parameter configurations which allows estimations in terms of the parameter spaces in which $\mathcal{C}$ - and $\mathcal{S}$-type reconfiguration events can be expected. Therefore, the Richardson number $R i=R a / P r \cdot R e^{2}$, which defines the relation of thermal to forced convection, is used as main distinction between the event types.

The Re-Ri parameter space based on Schmeling et al. (2013) is composed of areas with stable states (blue) as well as of states exhibiting reconfigurations of $\mathcal{S}$ - (red) and $\mathcal{C}$-type (green), see figure 3 . In order to generate different types of reconfiguration events, we applied the parameter sets $R i_{\mathcal{S}}=3.7, R a_{\mathcal{S}}=1.4 \times 10^{8}, R e_{\mathcal{S}}=0.7 \times 10^{4}$ and $R i_{\mathcal{C}}=$ $1.5, R a_{\mathcal{C}}=1.6 \times 10^{8}, R e_{\mathcal{C}}=1.2 \times 10^{4}$, which lie in the respective ranges. 


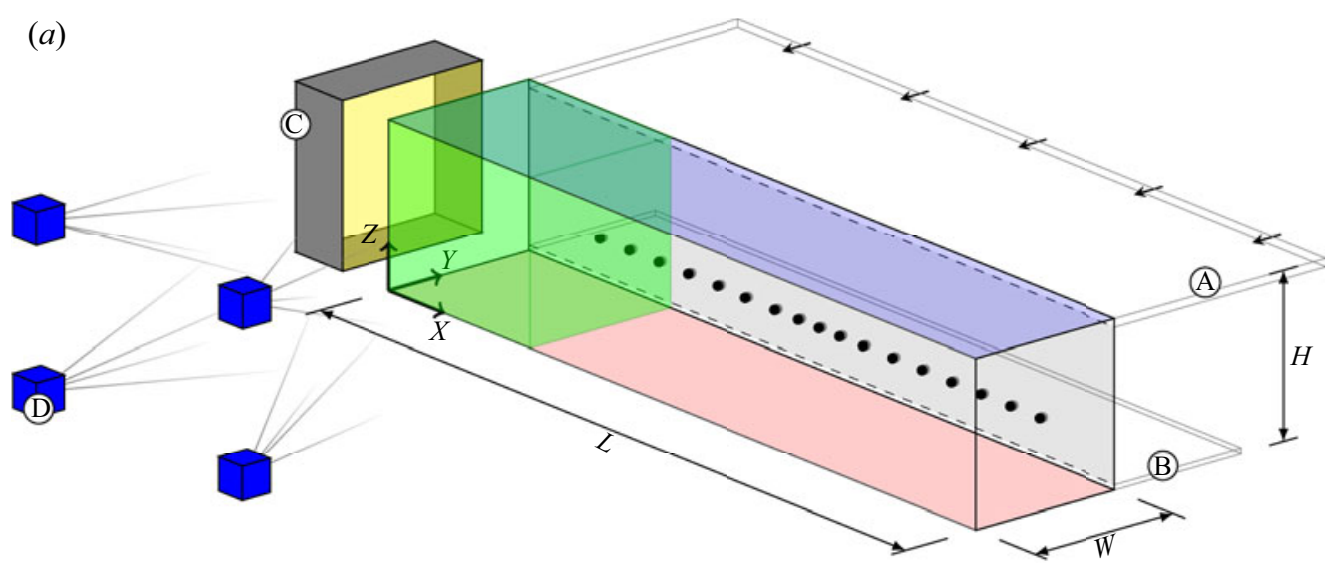

(b)

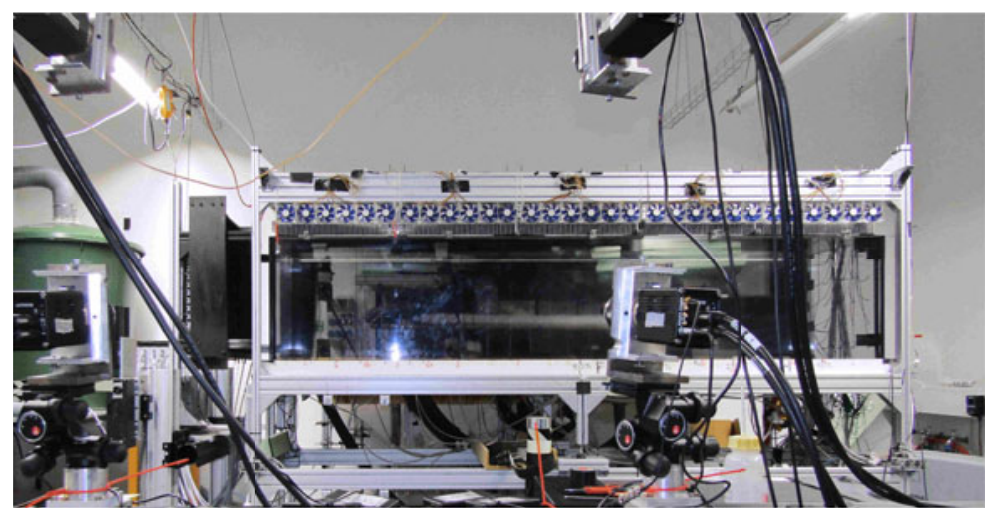

Figure 2. (a) Mixed convection sample with the used measurement set-up. A - inlet vent. B - outlet vent. C - LED light source. D - PIV camera system. The resulting PIV domain is highlighted in green. The positions of the temperature sensors near the rear wall are marked by black dots. (b) Photograph of the mixed convection sample.

It should be noted that the Archimedes number $A r$ was used as synonym for $R i$ in the publication of Schmeling et al. (2013).

\subsection{Measurement arrangement}

The measurement system was composed of a tomographic PIV set-up intended to investigate the evolution of flow structures and a temperature sensor array allowing a fast classification of the present flow state.

While Schmeling et al. (2013) used a temperature sensor array spread throughout the bulk sample for their investigations, we opted for a rear wall-bound arrangement to ensure optical accessibility of the PIV domain. Furthermore, wall-bound temperature measurements are a standard procedure for similar investigations in RBC (Brown \& Ahlers 2006; Funfschilling et al. 2008; Bai et al. 2016). Typically, the probes are circumferentially arranged at different heights. However, we reduced the sensor array exclusively to one line at the rear wall of the sample. This arrangement still provided sufficient information about the flow state as the forced flow fixes the up-welling fluid section to the rear wall for the investigated parameter range. The array comprised $17 \mathrm{Pt} 100$ resistive temperature sensors of precision class AA (IEC 2008). They were positioned at a height of $H / 4$ and 


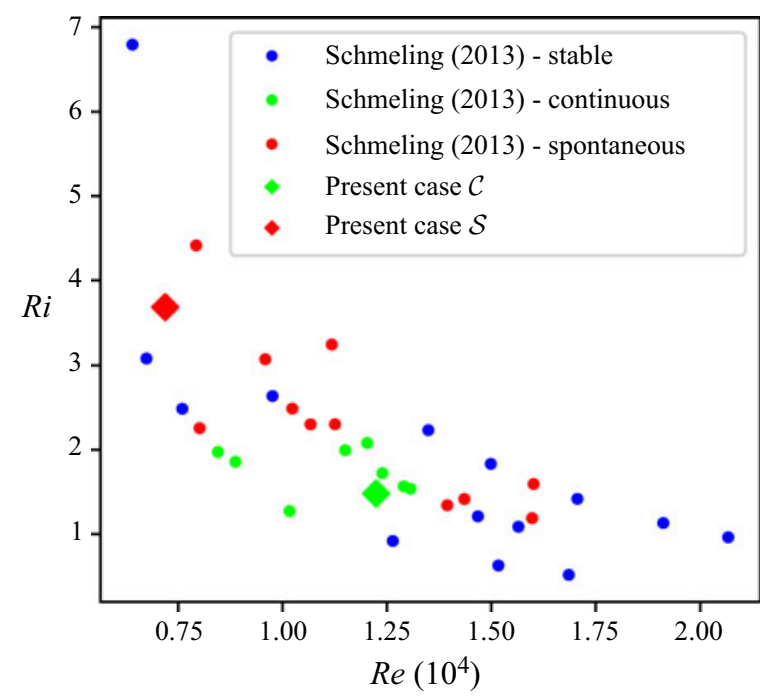

FIGURE 3. Parameter space with the classification determined by Schmeling et al. (2013): stable states as well as continuous and spontaneous reconfigurations are indicated by blue, green and red dots, respectively. Additionally, the cases studied in this work are marked by diamonds: the red diamond marks case $\mathcal{S}\left(R i=3.7, R a=1.4 \times 10^{8}, R e=0.7 \times 10^{4}\right)$ and the green one marks case $\mathcal{C}\left(R i=1.5, R a=1.6 \times 10^{8}, R e=1.2 \times 10^{4}\right)$.

in a distance of $W / 50$ to the rear wall, in accordance with Wessels et al. (2019). Their arrangement is indicated by the black dots in figure 2 . The sensor resistances were acquired by a scanning multimeter resulting in a measurement period of $\Delta t \approx 8.7 \mathrm{~s}$ for each single sensor. This frequency is of the order of the turnover frequency of the main convection roll. As the reconfiguration events occur on time scales at least one order of magnitude larger, this acquisition frequency was sufficient.

The measured temperature distribution is physically related to the global flow structure as $Y$-displacements of the convection roll are accompanied by changes in the temperature field. Therefore, changes of the rear-wall temperature distribution correspond to variations of the longitudinal distribution of LSCs (Niehaus et al. 2020).

Due to this interdependence, the temperature measurements also indicate the beginning of a flow reconfiguration event. Thus, we used them to trigger the PIV when a spontaneous reconfiguration occurred. This was necessary as the seeding precipitation during PIV would have affected the boundary conditions for longer ring buffer-based measurements. Details on the implementation of the trigger condition are described by Mommert et al. (2019).

In terms of the tomographic PIV set-up, we decided to measure the flow in the vicinity of the left sidewall in order to capture the expected structure formation process, which is part of the reconfiguration process described by Schmeling et al. (2013).

The corresponding PIV set-up is presented in figure 2. It shows the arrangement of the LED illumination (C), the camera system (D) and the measurement domain highlighted in green. To achieve particle images with sufficient contrast for PIV in a domain of this size, we chose the approach of LED-illuminated helium-filled soap bubbles similar to Kühn et al. (2012). Further information on the PIV system and its configuration for the measured cases can be found in table 1 . 


\section{Case $\mathcal{S} \quad$ Case $\mathcal{C}$}

Field of view

Interrogation volume

Observation distance

Recording medium

Recording lens

Illumination

Seeding material

Working aperture

Pulse delay

Imaging frequency

Measurement start
$X=0 \ldots 600 \mathrm{~mm}$

$Y=0 \ldots 500 \mathrm{~mm}$

$Z=0 \ldots 500 \mathrm{~mm}$

$40 \mathrm{~mm} \times 40 \mathrm{~mm} \times 40 \mathrm{~mm}$

$Y_{\text {camera }} \approx-1800 \mathrm{~mm}$

$4 \times$ pco.edge 5.5 CMOS camera

$4 \times$ Zeiss Planar $T^{*} 50 \mathrm{~mm} f / 1.4$

$15 \times 15$ white light LED array

Helium filled soap bubbles

$\begin{array}{cc}f / 4.8 & f / 5.6 \\ \tau_{\mathcal{S}}=12 \mathrm{~ms} & \tau_{\mathcal{C}}=10 \mathrm{~ms} \\ 0.91 \mathrm{~Hz} & 3.65 \mathrm{~Hz} \\ \text { perature triggered } & \text { Arbitrary }\end{array}$

TABLE 1. Recording parameters for tomographic PIV.

To ensure the required projection accuracy for the tomographic reconstruction of the acquired frames, we applied a volume self-calibration (Wieneke 2008) for each measured case in addition to the standard procedure of defining a mapping polynomial by capturing targets with known positions. Subsequently, tomographic reconstructions were performed using the simultaneous multiplicative algebraic reconstruction technique (Mishra, Muralidhar \& Munshi 1999). The velocity vectors were then determined by a three-dimensional cross-correlations. Except for minor changes, the algorithm of Kühn et al. (2011) was used for these procedures. Outlier velocity vectors were replaced after identifying them in two steps: First, all vectors with an unphysically high magnitude $\|\boldsymbol{u}\| \geq 1.6 v_{\text {in }}$ or low PIV correlation coefficient $r_{3 D}<0.2$ were marked. Second, further vectors of the remaining unmarked vectors were marked by means of the universal outlier detection (Westerweel \& Scarano 2005). Afterwards, all marked vectors were replaced by an interpolation between valid neighbouring vectors as methods like POD require gapless data. The number of outliers replaced in this way amounted to approximately $10 \%$ with the outliers being randomly distributed in the measurement volume.

\section{Proper orthogonal decomposition}

The characteristics of the different reconfiguration types were investigated by performing a POD analysis in order to determine the flow's coherent structures and their manifestation over the course of time in $\S 4$.

For the POD, each velocity vector field is reshaped into a single-state column vector $\boldsymbol{u}_{t}=\left[u_{X,(1,1,1)}, u_{Y,(1,1,1)}, u_{Z,(1,1,1)}, \ldots, u_{Z,(I, J, K)}\right]$. In this context, $I, J$ and $K$ are the number of grid points along the coordinate axes of the PIV domain.

Subsequently, the method of snapshots (Sirovich 1987) is applied to achieve the decomposition of these vectors into $k$ hierarchical modes $\boldsymbol{\phi}_{k}$ and the respective time coefficients $a_{k, t}$ as shown in (3.1)

$$
\boldsymbol{u}_{t}=\sum_{k} a_{k, t} \boldsymbol{\phi}_{k}
$$


Therefore, the series of $N$ discrete measurements in form of $\boldsymbol{u}_{t}$ are merged into the state matrix $\boldsymbol{U}$

$$
\boldsymbol{U}=\left[\begin{array}{cccc}
\mid & \mid & & \mid \\
\boldsymbol{u}_{1} & \boldsymbol{u}_{2} & \ldots & \boldsymbol{u}_{N} \\
\mid & \mid & & \mid
\end{array}\right]
$$

Next, the auto-correlation matrix $\boldsymbol{C}$ is calculated

$$
\boldsymbol{C}=\boldsymbol{U}^{\top} \boldsymbol{U}
$$

Solving the eigenvalue problem of (3.4) yields time coefficient vectors $\boldsymbol{a}_{k}=$ $\left[a_{k, 1}, a_{k, 2}, \ldots, a_{k, N}\right]$ and mode-related eigenvalues $\lambda_{k}$

$$
\boldsymbol{C} \boldsymbol{a}_{k}=\lambda_{k} \boldsymbol{a}_{k} .
$$

As each element of the auto-correlation matrix $\boldsymbol{C}$ is a product of two velocity components, the eigenvalues $\lambda_{k}$ represent a measure of the kinetic energy contained in a mode. Spatial representations $\phi_{k}$ of the latter can then be computed by

$$
\boldsymbol{\phi}_{k}=\boldsymbol{U} \boldsymbol{a}_{k} .
$$

For the interpretation of the modes, it is important to consider that translatory moving coherent structures are not extracted into single modes by the POD method. Instead, this method distributes the moving structure to a set of modes, similar to a Fourier decomposition, in order to reproduce the movements (Brunton \& Kutz 2019, pp. 396-397). Such a Fourier-like representation is related to a slow decay of the eigenvalues. As Schmeling et al. (2013) conjecture a translation of flow structures, the PODs have to be interpreted with particular care with regard to this issue.

Furthermore, it should be noted that the present PODs were based on the uncentred state matrices $\boldsymbol{U}$. This is of particular interest, as the first mode of an uncentred POD can be similar to the averaged field. That way, the POD also quantifies the energy contained in that structure.

\section{Results and discussion}

\subsection{Rear-wall temperature distribution}

The first part of the analysis focuses on the temperature data to establish a relation to previous studies and to gain first information about the time development of the reconfiguration processes including their consistency.

In order to identify the LSCs based on local temperatures, the evolution of the spatial interpolation between the rear-wall sensors is displayed in figure 4 for the considered cases $\mathcal{S}$ and $\mathcal{C}$. It displays the respective time-averaged temperature distributions on the left side: while the two hot spots $(L / 4,3 L / 4)$ of the stable periods become visible for case $\mathcal{S}$, the distribution of case $\mathcal{C}$ shows no clear structures as this case did not exhibit stable periods.

The evolutions of the associated temperature deviations on the right of figure 4 reveal a number of hot spots (HS) mainly moving from the left to the right $X$-positions for both cases. During the reconfiguration events of case $\mathcal{S}$, the deviations become more intense, as the structure of the stable periods is already imprinted on its mean distribution.

The main distinctive features of the cases are the time scales of the hot spots' dynamics and the number of implied LSCs. To quantify the latter, we consider the following: each hot spot is associated with up-welling warm fluid, whereas the compensating colder flow 

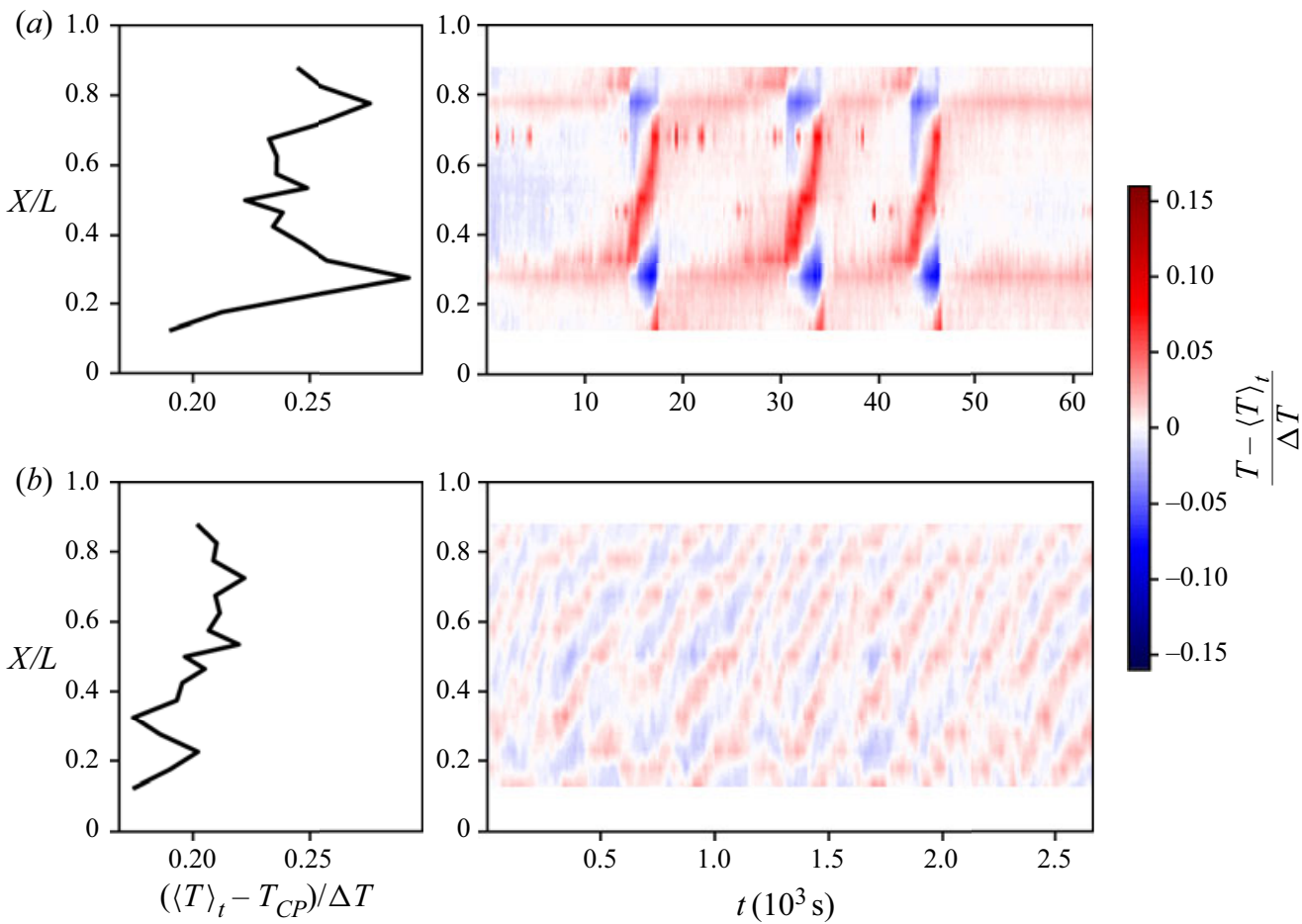

FIGURE 4. Time-averaged temperature distributions for the rear-wall sensor array (left) and the time evolutions of the deviations from the mean value (right) shown for both cases $\mathcal{S}(a)$ and $\mathcal{C}(b)$.

regions are located on the hot spot's sides and expressed as cold patches in figure 4. For an initial guess, the wavenumber $w$, here defined by the number of LSCs, was calculated by the distances $\Delta X$ between a hot and a cold spot (CS) or two hot or cold spots:

$$
w \approx \frac{L}{\Delta X_{H S-C S}} \approx \frac{2 L}{\Delta X_{H S-H S}} \approx \frac{2 L}{\Delta X_{C S-C S}} .
$$

On this basis, we estimated $w_{\mathcal{S}}=4$ and $w_{\mathcal{C}}=8$ for the respective cases. These numbers correspond to the findings of Westhoff (2012, pp. 54-59), who also found these two wavenumbers associated with high and low $R i$ in a study of turbulent mixed convection cases with cases with similar $R a$.

In order to gain temporal information on this matter, the number of LSCs was determined over the course of time by applying a cosine-based curve fit (4.2) to the instantaneous temperature distributions $T_{t}(X)$. The fit parameters were determined by minimising the sum the residuals' squares with Newton's method. As the temporal resolution was sufficient to continuously resolve transformation processes, the established fit parameters of the previous instant were used as starting conditions for the succeeding time step. Since the reconfiguration process is characterised by decaying and emerging LSCs (Schmeling et al. 2013), we focused on the cosine summands next to the suggested wavenumbers $w_{\mathcal{S}}=4$ and $w_{\mathcal{C}}=8$. Besides the number of LSCs, further information about the states is contained in the sign of $A_{n}$. Thereby, a positive sign corresponds to a hot spot 


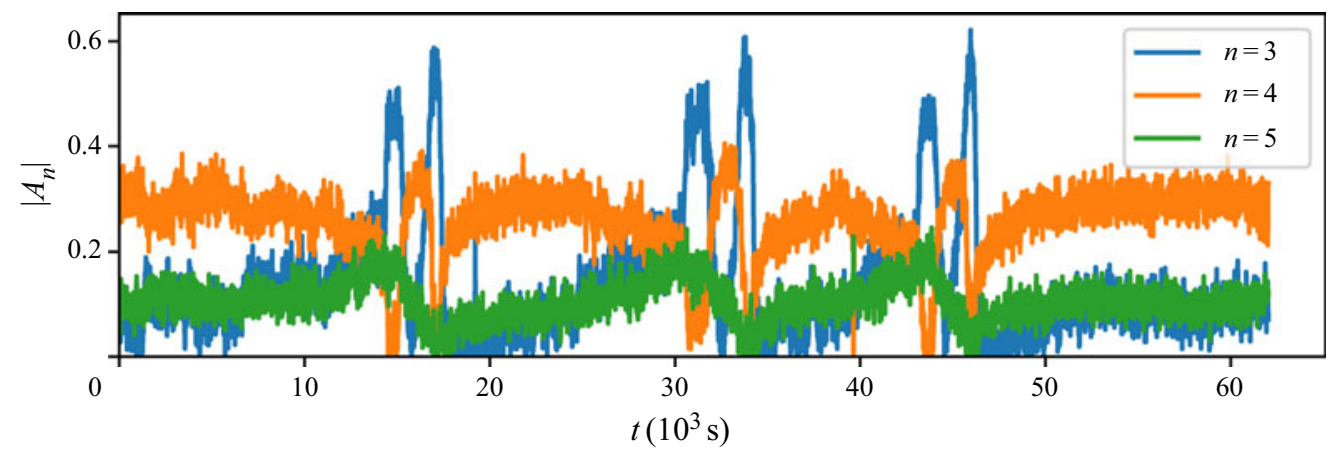

FIgURE 5. Temporal development of the absolute values of the cosine fit amplitudes $\left|A_{n}\right|$ implying the number of LSCs in the sample for case $\mathcal{S}$.

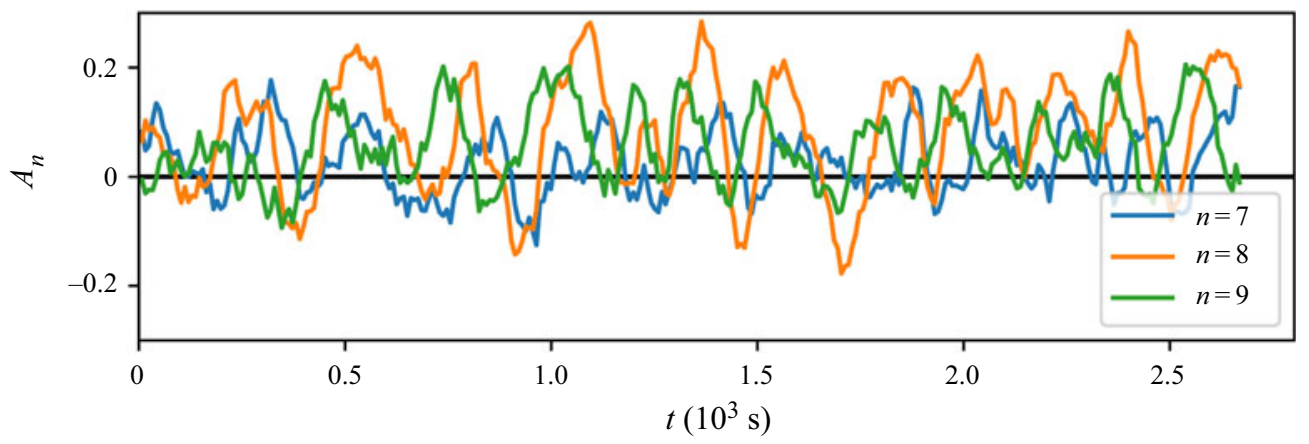

FIgURE 6. Temporal development of the cosine fit amplitudes $A_{n}$ implying the number of LSCs in the sample for case $\mathcal{C}$.

at the left sidewall.

$$
f_{T}(X)=\sum_{n=w-1}^{w+1}\left(A_{n} \cos \left(\frac{n \pi X}{L}\right)\right)+T_{0} .
$$

For both cases, the amplitudes $A_{n}$ are plotted in figures 5 and 6, respectively. Regarding case $\mathcal{S}$, the absolute amplitudes show that a reconfiguration event consists of multiple changes between 4 and 3 LSC states, while $\left|A_{5}\right|$ never dominates. However, during the multiple hour long periods of stability, $\left|A_{3}\right|$ and $\left|A_{5}\right|$ are steadily rising. Similar rises can be observed for $\left|A_{3}\right|$ during the short 4 LSC periods of the reconfiguration events.

This shows that case $\mathcal{S}$ is characterised by a continuous reconfiguration process although it exhibits distinct events. The existence of a quasi-stable period between the events indicates that there is a preferred flow state with four LSCs.

Figure 7 allows us to describe the process in more detail as it depicts the phase space of the signed amplitudes $A_{n}$. It visualises the quasi-stable periods of the preferred state as an accumulation of data points representing a 4 LSC state with $A_{4}<0$. This means that the cold areas appear at the sides of the sample for most of the time, which was also found to be the stable configuration for a wide $R a$-range in numerical simulations of RBC (Kaczorowski \& Wagner 2009).

As the depiction covers three events which cannot be distinguished, it shows that they proceed as a consistent process: the preferred 4 LSC state is followed by a 3 LSC state 


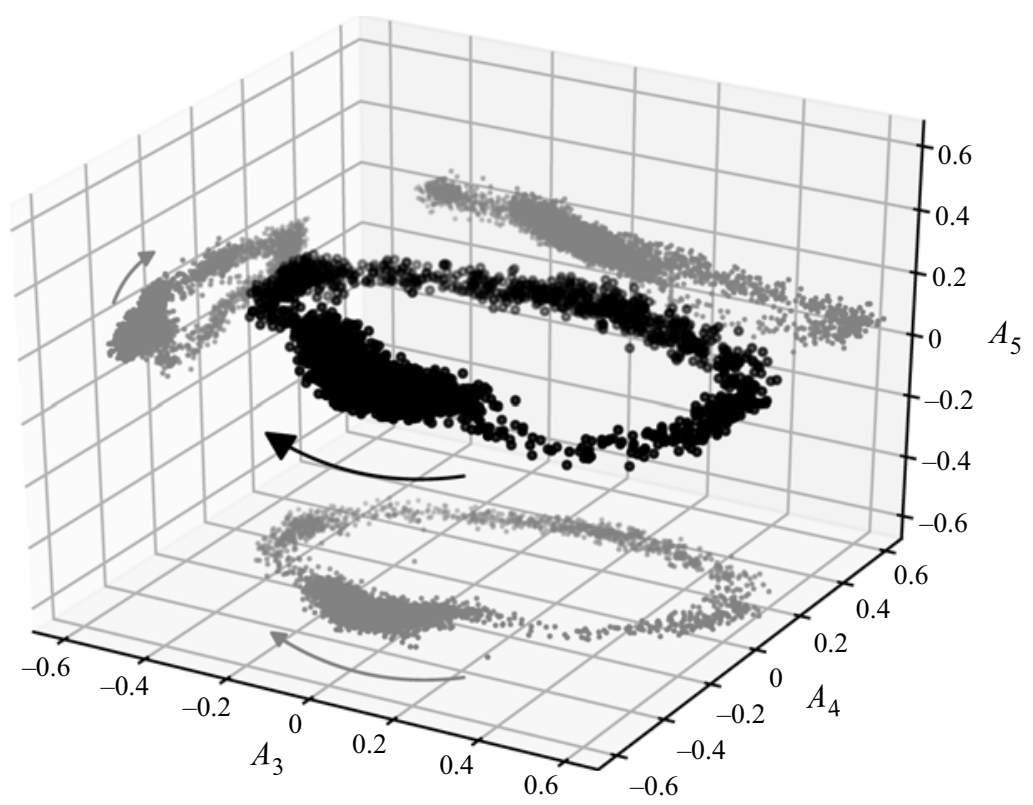

FIGURE 7. Phase space of the fit amplitudes $A_{n}$ of case $\mathcal{S}$ allowing us to determine the exact orientation by the means of the amplitudes' sign.

with a cold left side $\left(A_{3}<0\right)$. The flow then passes through a 4 LSC state with warm sides before the circle is closed by traversing a 3 LSC state with a warm left side. Such a switching between states with different retention times was also observed by Xie, Ding \& Xia (2018) in an annular RBC sample. In contrast to their study, the two main states in the present investigation exhibit the same wavenumber and only their transition is predominated by a state of a lower wavenumber.

During the whole process, $A_{5}$ plays only a minor role. This is in good agreement with the observations of Schmeling et al. (2013), which describe the same cycle process with an LSC decay on the right side followed by the formation of a new LSC on the left side. Regarding the origin of these events, the slow rise of $\left|A_{3}\right|$ and $\left|A_{5}\right|$ during the quasi-stable phase also corresponds to the idea of a heat or momentum accumulation mechanism. That could further mean that the distinct spontaneous events equal the passing of an accumulation threshold accompanied by the release of the earlier accumulations similar to Sugiyama et al. (2010) or Wang et al. (2018).

Regarding case $\mathcal{C}$, the $A_{n}$ evolution shown in figure 6 reflects the continuous dynamics with a mean period of $t_{\mathcal{C}} \approx 215 \mathrm{~s}$. Our initial wavenumber guess is confirmed as the coefficient $A_{8}$ displays the highest amplitudes. Therefore, we define 8 LSCs as the underlying baseline for this case and use $A_{8}$ as an indicator for its dynamics. In contrast to case $\mathcal{S}$, the maximum amplitudes of $A_{n}$ are only half as large. This means that random turbulent fluctuations have a stronger influence and the reconfiguration process appears more chaotic. However, the following systematics are revealed: the intervals of sign change with positive gradient of $A_{8}$ display a positive $A_{9}$ as prevalent amplitude parameter. During a change of sign with negative gradient of $A_{8}$, a positive $A_{7}$ is prevalent.

This can be interpreted as follows: an 8 LSC state with cold sides transforms into an 8 LSC state with warm sides via the generation of a new counter-rotating LSC on the left side followed by the decay of the rightmost LSC. During the opposite transformation, the 


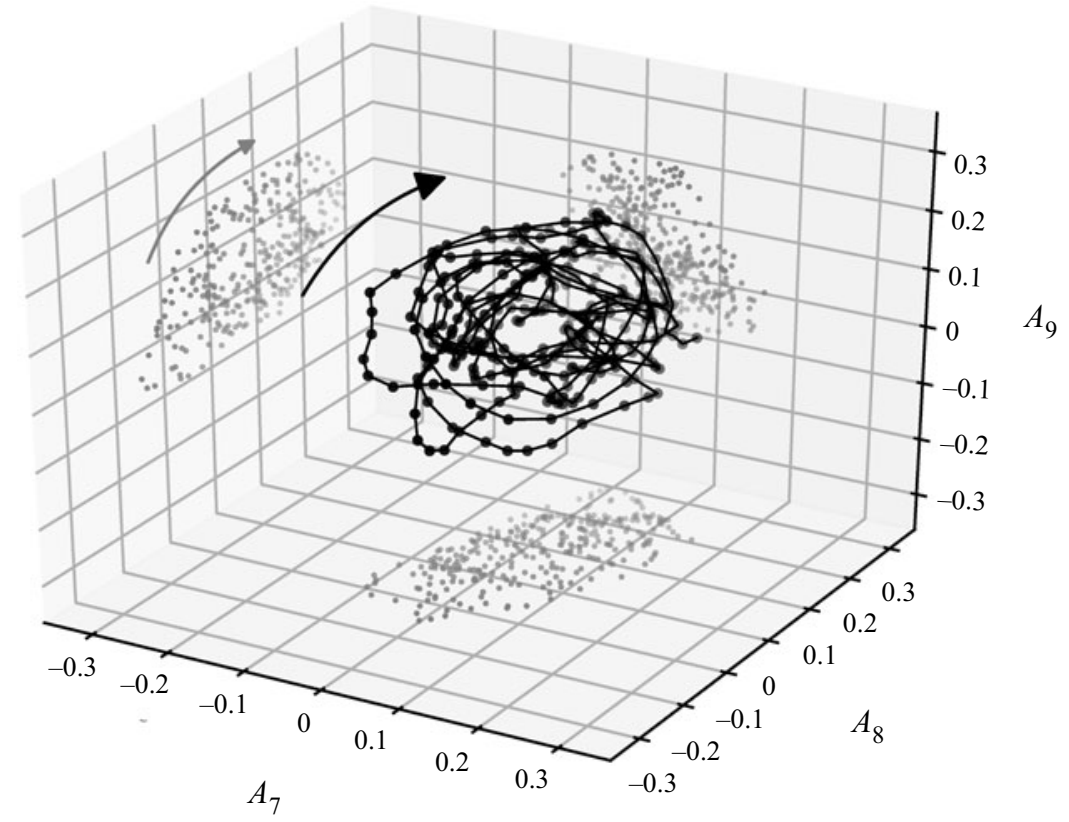

FIgURE 8. Phase space of the cosine fit amplitudes $A_{n}$ for case $\mathcal{C}$. The displayed dots are moving averages of $A_{n}$ over 3 data points.

decay on the right side takes place first and is succeeded by the formation of a new LSC on the left side. Further, the observations also reveal a bias of case $\mathcal{C}$ towards positive values of $A_{n}$, while for case $\mathcal{S}$ the differently signed extents of $A_{3}$ and $A_{4}$ were almost symmetric but varied in duration. The fact that this manifestation of the bias does not create quasi-stable states indicates that these reconfigurations are driven by different forces. Hence, the share of forced convection remains as the main driver for the reconfigurations case $\mathcal{C}$. This idea is followed up by the analyses of $\S 4.3$.

Figure 8 also confirms the consistency of this case as a prevalent orbit exists in the phase space of case $\mathcal{C}$. In contrast to case $\mathcal{S}$, the orbit is tilted inside the three-dimensional (3-D) phase space, as both, $A_{7}$ and $A_{9}$, play an important role.

Despite possible differences regarding the driving forces, the findings of the temperature measurements indicate that the reconfiguration mechanisms of both cases are based on the translation of flow structures in the sample accompanied by the generation and decay of LSCs at the sidewalls. This result corresponds to the conclusions drawn by Schmeling et al. (2013). To test this model concept, the results of 3-D PIV measurements performed during the presented time series are addressed in the next sections.

\subsection{Velocity fields of spontaneous events}

For assessing flow field information of an event of type $\mathcal{S}$, we used a temperature-based trigger, see \$2.2. Especially, a condition checking for a change in the relation of two local temperatures between the previous $(t-\Delta t)$ and latest $(t)$ measurements was found to be an indicator for the onset of an event in preliminary tests (Mommert et al. 2019), see $(4.3 a, b)$. Its instant of occurrence is also marked by a circle in figure $9(a)$, which depicts the evolution of the respective temperature signals. Additionally, the colour-coded 
background indicates periods of $\left|A_{3}\right|$ or $\left|A_{4}\right|$ dominance based on the cosine fits.

$$
\begin{aligned}
& T(X=0.275 L, t-\Delta t)>T(X=0.425 L, t-\Delta t) \quad \text { and } \\
& T(X=0.275 L, t) \leq T(X=0.425 L, t) .
\end{aligned}
$$

Limited by the delay for achieving a sufficiently high seeding density, we acquired flow data for the time span marked by a blue bar in figure $9(a)$, which covers approximately $75 \%$ of the event. Exemplary flow fields of this time span are presented in figure $9(b)$. They comprise velocity vectors in three cross-sections and streamlines in regions of relatively low velocities $\left(\|\boldsymbol{u}\| / v_{\text {in }}<0.2\right)$ to visualise the convection roll structure in the domain.

Regarding the temperature analysis, the first observed state (I) represents the beginning of the transition from a 3 LSC configuration with prevalent cold temperatures at the left sidewall to four LSCs with a 'warm sidewall'. At this instant, the streamlines reflect a single convection roll core with a diagonal alignment characterised by a displacement towards the front and top on the left side and rear and bottom on the right side. We assign this instant already to the onset of a 4 LSC state, as instants II and III display an intensified version of this alignment of the main roll core. At the same time, first traces of the formation of a secondary structure can be observed for instant II: at $X \approx L / 8$, another roll core branches out on the rear side of the main roll and extends to the left sidewall where it curves towards a $Y$-parallel alignment. This formation prevails throughout the dominance of $\left|A_{4}\right|$ as velocity field III represents a similar formation.

At time instant IV, the onset of the $\left|A_{3}\right|$-dominant interval, the secondary structure vanishes and the diagonal displacement of the main roll from its central location is reduced. Thus, it can be considered as central and straight. With $\left|A_{4}\right|$ becoming dominant again at instant $\mathrm{V}$, the main roll realigns with the inverse diagonal (left rear to upper right front) which also entails a secondary roll branch. For this alignment, the secondary structure branches also from the rear side of the main roll at $X \approx L / 8$ and stretches to $X \approx L / 4$ corresponding to the hot spot location at the rear wall. There, it aligns parallel to the $Y$-axis. Velocity field VI shows that this branch alignment is prevalent for the 4 LSC configuration observed during the remainder of the measurement period.

Especially, the occurrence of a secondary roll branch raises further questions with regard to possible links to corner roll-driven reversals in RBC; see Sugiyama et al. (2010) and Soucasse et al. (2019). For a stable mixed convection case, Kühn et al. (2012) also found $Y$-parallel vortical structures, which occurred in pairs and were otherwise similar to the structure of the secondary branches. Therefore, we extrapolate that a mirrored secondary branch outside the PIV domain exists for fields like V or VI, see figure $9(c)$. Regarding their dynamic behaviour, we observed the decay of these branches at the onset of the reconfiguration rather than a growth. This rules out a reversal process analogous to $\mathrm{RBC}$ in quasi two-dimensional samples.

In the following our observations will be summarised and evaluated. In terms of the temperature analysis, we expected the following behaviour of the main convection roll: during $\left|A_{3}\right|$-dominance, the diagonal segments stretch to fill the space along the $X$-axis. Under this assumption, the secondary branches persist and move corresponding to the hot spots over the course of a reconfiguration; compare with figure 4 . However, we found that the flow structures occurring in the monitored part of the sample during $\left|A_{3}\right|$-dominance reflect a transition state without strong diagonal displacements or secondary roll branches; see instant IV. While a translational propagation of the secondary structures is plausible for more $X$-central regions of the sample, our observations contradict the translational model regarding the dynamics of the leftmost LSC. Rather, the motion of this segment of the main convection roll is described by the switch of orientation of the observed roll 
(a)

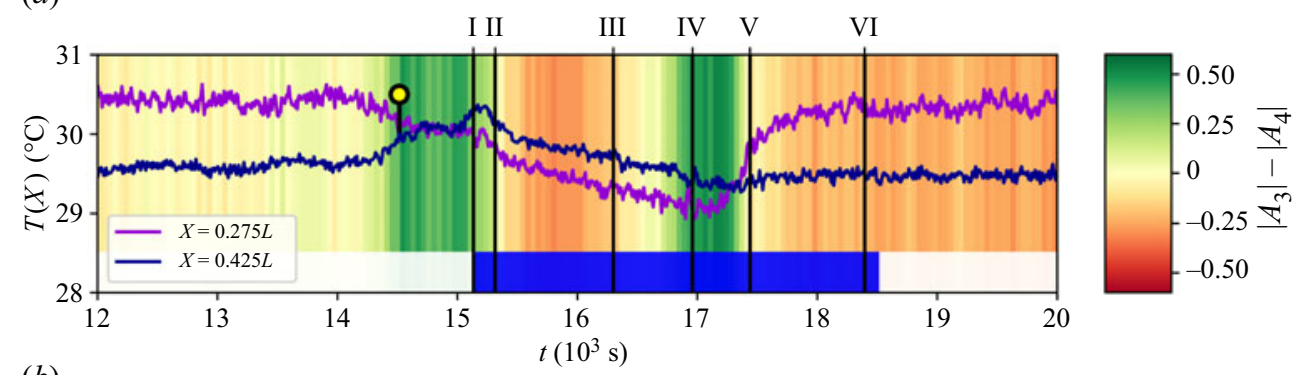

(b)
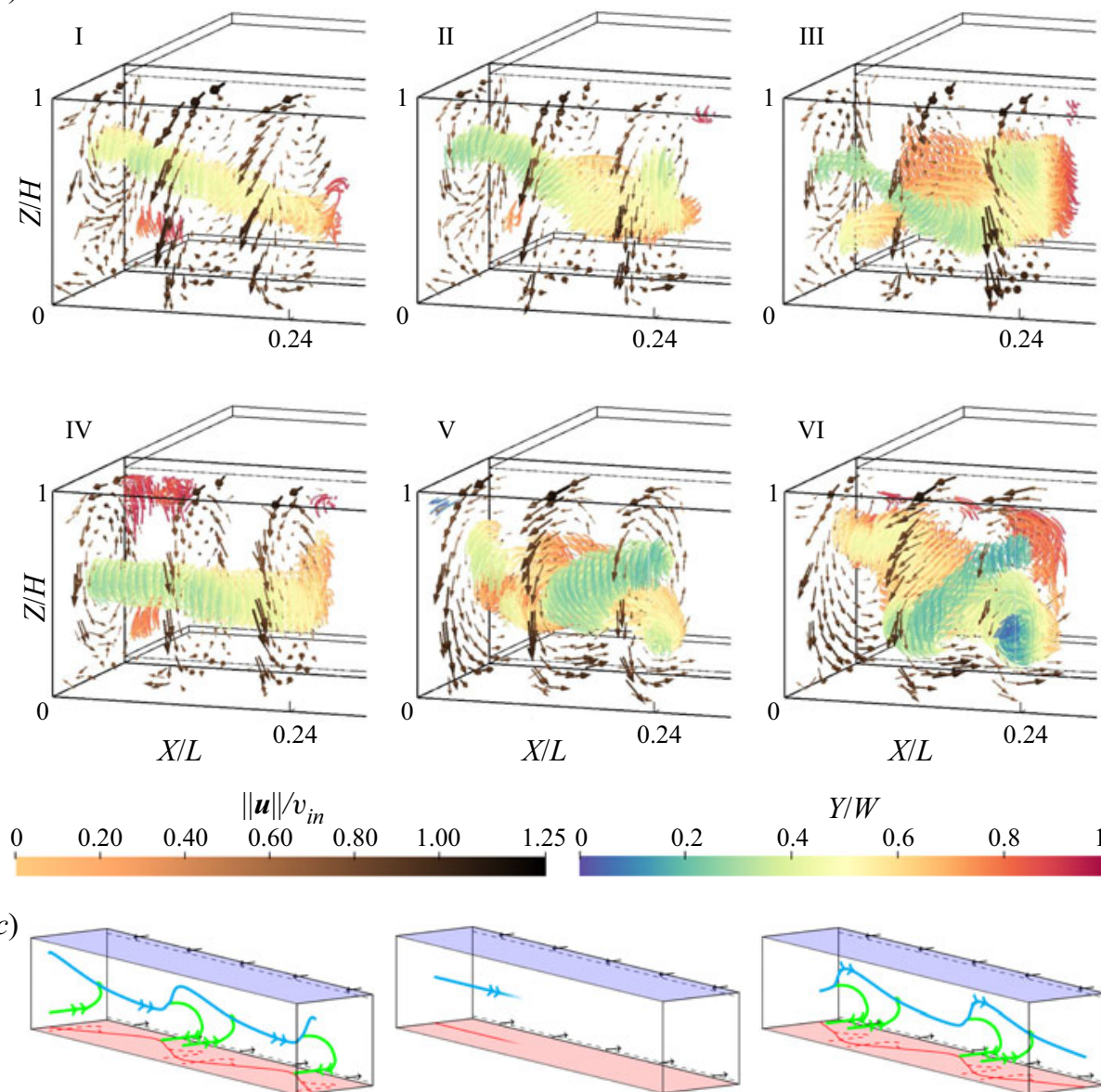

Idealised transition (IV)

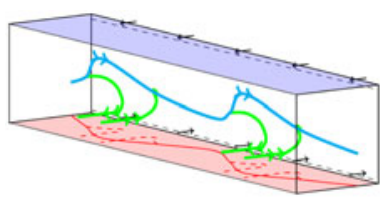

Extrapolation for V, VI

FIGURE 9. (a) Temperature time series recorded by selected sensors over the course of a reconfiguration event. The interval for which velocity fields were measured is marked by a blue bar. (b) Velocity fields for 6 points in time, which are indicated in the temperature series. The fields are represented by vectors in 3 cross-sections and streamlines in areas of $\|\boldsymbol{u}\| / v_{\text {in }}<0.2$ reflecting the convection roll core. The displayed fields are short-time averaged over 9 frames. Supplementary movie 1, available at https://doi.org/10.1017/jfm.2020.705, shows the complete time series. (c) Sketches of the extrapolated or idealised flow structures. The main convection roll and the secondary branch are marked in blue and green, respectively. They are projected onto the bottom plate in red. 
(a)
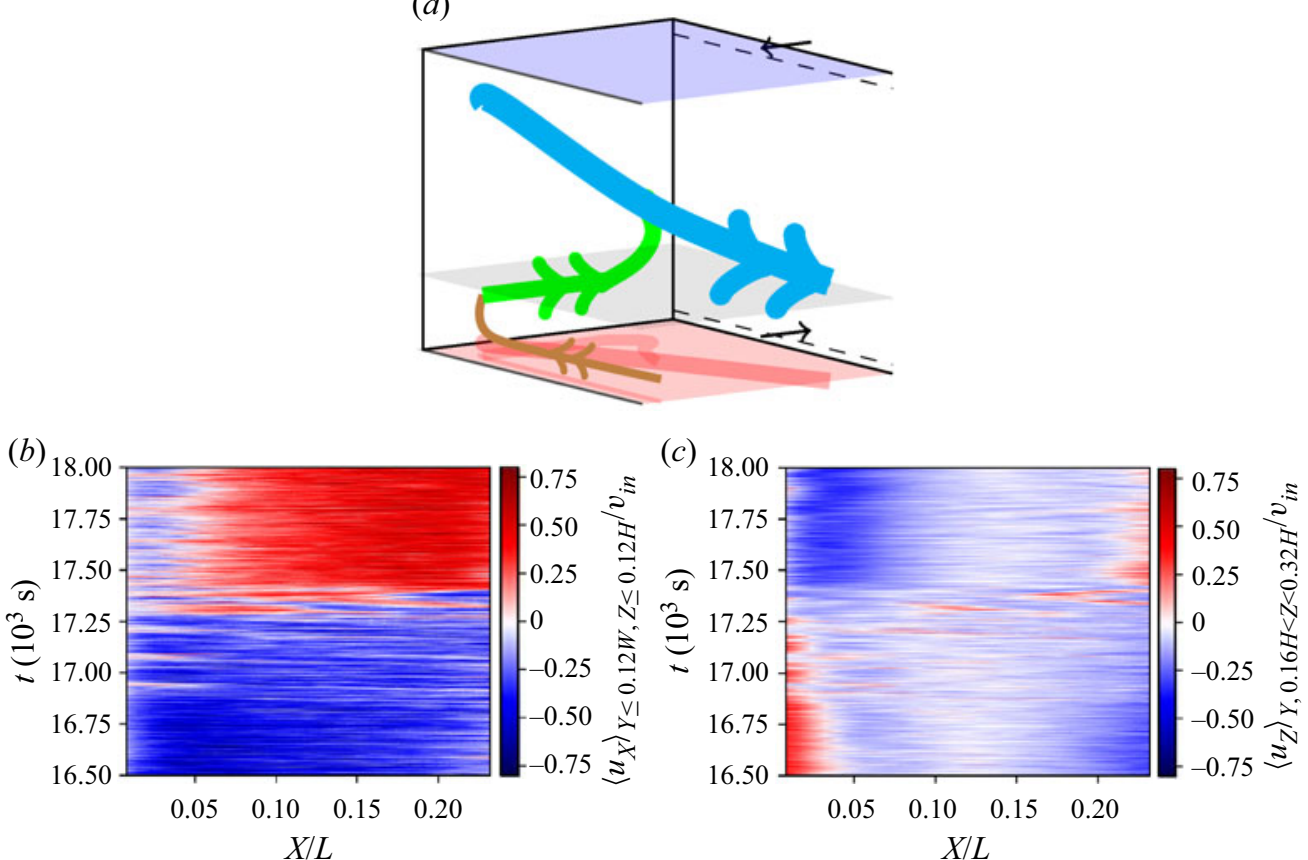

FIGURE 10. (a) Vortex system represented by angular momentum arrows in the PIV region of the sample. Blue: main convection roll. Green: secondary roll branch. Brown: front bottom edge vortex. Grey: control region for plume event analysis. (b) $X$ velocity component averaged over the region of the edge vortex. (c) $Z$ velocity component averaged over the region of the control plane.

segment around a pivot at $X \approx L / 8$, which we will refer to as switching. This raises the question why figure 4 displays a translating hot spot in the region overlapping the PIV domain, where no secondary structure was observed during the transition (see figure 9c).

The reason for these observations is that the position of the temperature sensor array leads to the acquisition of temperature footprints related to local structures occurring during the reconfiguration events. These structures are plumes which originate from the front bottom edge vortex of the sample. Figure 10 shows the position of the edge vortex in relation to the main convection roll and its secondary roll branch. In order to describe the mechanism responsible for a reconfiguration event, the longitudinal velocity component in the region of the edge vortex is depicted in figure 10(b). Similarly, figure $10(c)$ shows the spanwise-averaged vertical velocity component in the control region drawn in a).

Regarding the longitudinal flow within the edge vortex, it is evident that this vortex transports fluids towards the secondary roll branch. However, this mechanism changes its direction, when the flow reconfigures itself $\left(17.0 \times 10^{3} \leq t \leq 17.5 \times 10^{3}\right)$. Similar to 2-D RBC (Sugiyama et al. 2010), the edge vortex gathers heat from the bottom plate. Due to the small size of this circulation, the heat can hardly dissipate through the boundaries of the vortex. Therefore, the longitudinal convection within the vortex plays a major role when it comes to transporting the accumulated heat. Apparently, a reconfiguration occurs when this longitudinal transport is interrupted and switches its direction - most likely due to saturation effects. At the same time, the heat is not sufficiently removed 

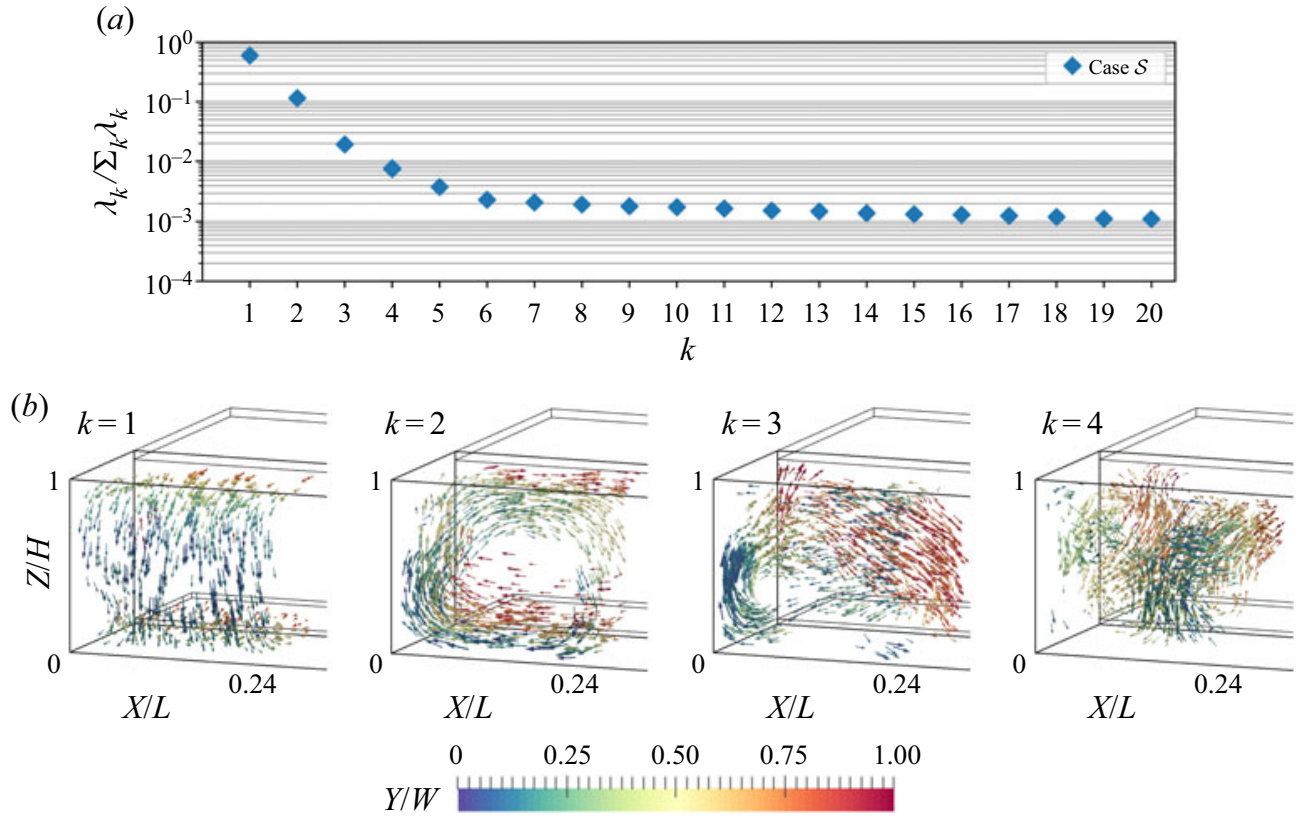

FIgURE 11. Eigenvalue distribution for the POD of case $\mathcal{S}(a)$ and the four most prevalent modes normalised by the square root of the respective eigenvalue $(b)$. Their structure is represented by the vectors of the highest magnitude. Supplementary movie 2 contains tracking shots of the displayed structures.

from the edge vortex, which leads to multiple eruptions of plumes. The latter disturb the main roll and therefore promote its reorientation (see Huang \& Xia 2016). Evidence for these plume eruptions is provided by the averaged vertical velocity component in a control plane displayed in figure $10(c)$. It shows that footprints of these plumes occur when the longitudinal transport of the edge vortex switches direction. Therefore, the translation of hot spots observed in the temperature distributions of the rear wall is associated with events of local plume eruptions.

In the meantime, the macroscopic flow structure follows certain maximum and minimum principles: the two diagonal arrangements of the LSC represent potential minima. Regarding the changes between these states, the potential barrier for a switching appears lower than for a translation, as the latter would require the generation of a very narrow diagonal convection roll segment at the sidewall.

In order to gain insight into the macroscopic processes behind the switching of this roll segment, we investigated the underlying coherent structures. Hence, a POD was conducted with the instantaneous velocity fields. Applying the approach of Podvin \& Sergent (2015), representations of the structure of the most prevalent modes and the respective eigenvalue distribution are presented in figure 11 for this case.

Most remarkably the first POD mode is represented by a vector field shaped like a longitudinal convection roll similar to the one of pure forced convection (Westhoff et al. 2010; Kühn et al. 2012); see figure 11(b). Although the Richardson number $R i=3.7$ indicates that a buoyancy dominated flow exists, this forced convection mode acquires $60 \%$ of the kinetic energy. However, it will be shown that the flow dynamics is mainly represented by the second mode, which we assign to the contribution of thermal convection. This is due to the fact that the large-scale circulation of the second mode 


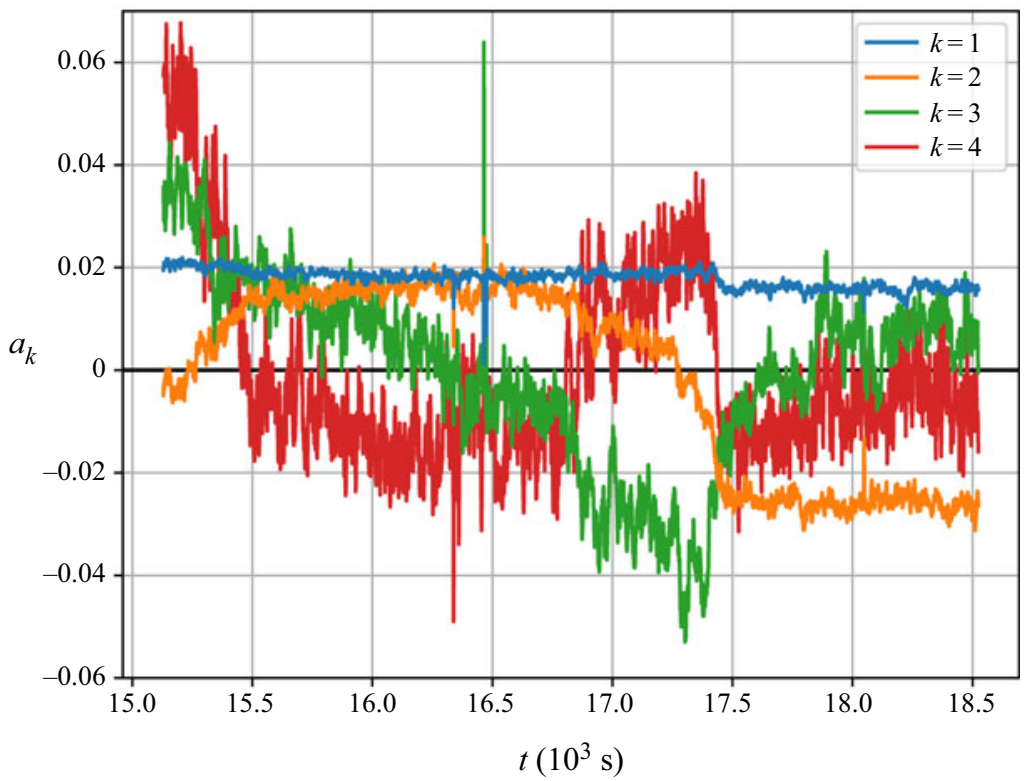

FIgURE 12. Time development coefficients for the first four POD modes as presented in figure 11.

is aligned in $Y$-direction. This agrees with the alignment obtained for pure thermal convection, i.e. RBC, in samples with the same aspect ratios (Kaczorowski \& Wagner 2009; Podvin \& Sergent 2012). The fraction of the second mode's eigenvalue amounts to $11.5 \%$. With both $X$ - and $Y$-angular momenta covered by the first two modes, these allow us to reconstruct the convection roll's diagonal alignment and rotation in the central $X Y$-plane. Although only $2 \%$ and $1 \%$ of the overall energy is contained in the following modes, their influence on the reconfiguration process cannot be excluded: these modes also mark the transition from large-scale contributions to the coverage of localised structures. The third mode comprises not only a large-scale circulation aligned in $Z$-direction but also a strong contribution to an upward flow near the front left vertical edge of the sample. Mode 4 is even more localised, with a downward flow in the front part of the sample, which makes the strongest contribution at $X \approx 0.14 L$. Regarding the considerations on PODs in terms of translating structures, it is certain that the present POD does not represent a translation-dominated process, since the eigenvalues would decline significantly slower in that case (Brunton \& Kutz 2019, pp. 396-397). Further, modes 3 and 4 do not display structures comparable to Fourier modes, that means multiple rolls with a $Y$-angular momentum. Instead, they show more localised and complex structures as the example of mode 4 shows. However, no further modes will be presented due to the advancing decline of their eigenvalues and therefore decreased contribution to the flow.

Another reason for this can be deduced from figure 12 displaying the temporal development of the modes: higher modes cover structures associated with small(er)-scale turbulence and increasing measurement noise which results in the time evolution becoming noisier and harder to interpret.

However, the presented modes allow us to shed more light on the flow processes during a reconfiguration event: the first mode's coefficient remains constant until $t \approx 17.5 \times 10^{3} \mathrm{~s}$, then it drops from 0.02 to 0.016 . We explain this drop as an artefact of the incomplete 
reconfiguration process as input of the POD. It can also be retraced in an imperfection of the longitudinal convection roll visible at the left vertical edge of the vector plot $k=1$ in figure 11. However, since the change of this coefficient is small compared to the dynamic of the other coefficients, the reconfiguration is captured sufficiently by the POD.

The coefficient of mode 2 crosses zero at $t \approx 15.2 \times 10^{3} \mathrm{~s}$ and rises to 0.02 where it remains stable during the time span $15.75 \times 10^{3} \mathrm{~s} \leq t \leq 16.75 \times 10^{3} \mathrm{~s}$. Afterwards, it falls with increasing rates to a value of -0.025 and levels off for the remainder of the time series. As for the influence on the flow, the coefficient's changes of sign at $t \approx 15.2 \times 10^{3} \mathrm{~s}$ and $t \approx 17.3 \times 10^{3} \mathrm{~s}$ imply that the main thermally induced structure reverses twice during a reconfiguration event. Interpreting this in the context of super-positioned angular momenta, a reversing second mode reflects a change in the diagonal alignment of the main convection roll by rotation (see Soucasse et al. 2019; Vasiliev et al. 2019).

This poses the following question: how do the buoyancy-induced coherent structures behave in comparison with the LSCs of RBC?

A similarity to the cessations observed in cylindrical RBC (Brown \& Ahlers 2006) is indicated by the two zero crossings in the time course of the coefficient of the second mode. These two zero crossings reflect that the main structure driven by the buoyancy force ceases to exist during reconfiguration events. The cessation process is, like other Poisson processes in cubic RBC (Bai et al. 2016), based on the breaking of a potential barrier by turbulent fluctuations. However, taking the remaining modes 3 and 4 into account contradicts the explanation of random fluctuations as a cause for the reversals of the present case: in particular, the coefficients of mode 3 and 4 display increased absolute values during the sign changes of mode 2 and lower ones during the level intervals of mode 2. This means that momentum is transferred between the coherent structures of mode 2 and the two following modes. Thus, this exchange resembles a coordinated process rather than random fluctuations as it proceeds for both sign changes of mode 2 .

Further investigation of mode 3 in figure 12, reveals that this mode approximates the change rate of mode 2 . As mode 3 is associated with an angular momentum in the $Z$-direction, it might represent a azimuthal motion which drives the reconfiguration (see Vasiliev et al. 2019). However, the consideration of the sign of $a_{3}$ yields an azimuthal flow contrary to the one expected from the evolution of the flow structure. That means the observed reconfigurations are not driven by azimuthal flow. Rather, the $Z$-oriented rotation of mode 3 adds another, vertical angular momentum component to the model describing the diagonally aligned convection roll with the first two modes. This way, mode 3 mainly represents an additional vertical tilt of the convection roll core. Tangible descriptions of its effect are given by the depiction of the reconstructed roll cores in figure 13: the addition of mode 3 allows the reconstruction of a stronger elevation of the roll core next to the sidewall for time step II. On the contrary, the correct central core position at time step IV is also achieved by including mode 3 .

Furthermore, the time coefficient of mode 3 falls constantly in the period $15.75 \times$ $10^{3} \mathrm{~s} \leq t \leq 16.75 \times 10^{3} \mathrm{~s}$, in which the direct observation of the flow fields revealed no significant transition process. This again underlines the presence of a accumulation and release reversal mechanism (Wang et al. 2018). In detail, the kinetic energy accumulated in the coherent structures of mode 3 is released and transferred to mode 2 rather quickly after a critical level was reached $\left(t \approx 17.4 \times 10^{3} \mathrm{~s}\right)$.

Further indication for this kind of process is provided by mode 4: it keeps fluctuating around -0.01 for most of the time but shows elevated values surpassing 0.03 while the large-scale flow structure is being transformed. Spatially, this mode has the strongest contributions at $X \approx 0.14 L$, see figure 11 . Hence, we associate its flow structure with the 'release' which triggers the progression of the reconfiguration. 

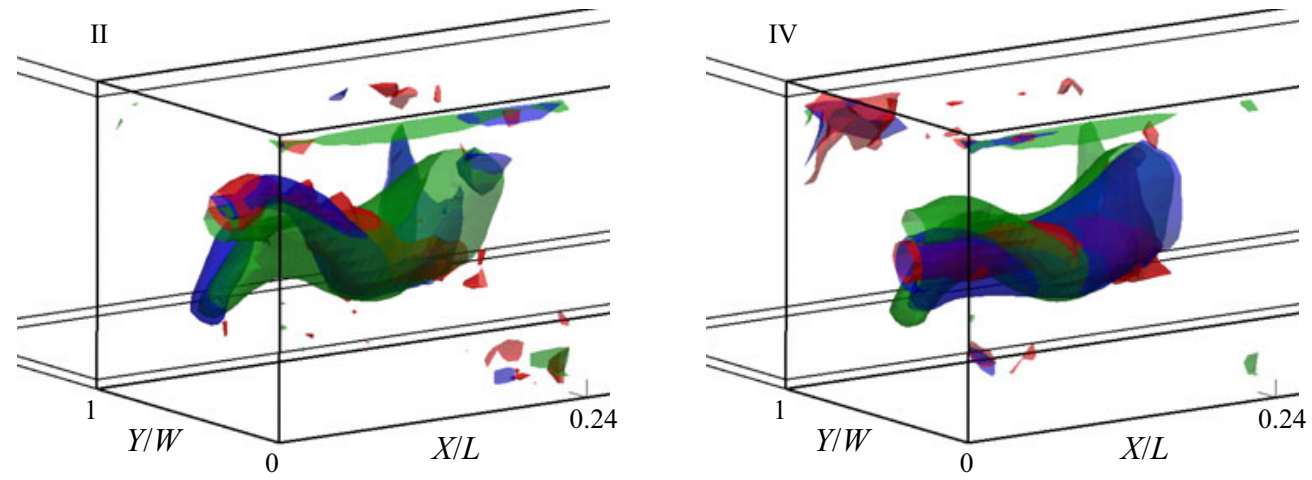

FIGURE 13. The $\|\boldsymbol{u}\|=v_{\text {in }} / 8$ isosurfaces representing the convection roll core for the short-time average PIV fields (red) and the POD reconstructions with the modes $k \leq 2$ (green) and $k \leq 3$ (blue) for the times II and IV from figure 9(a). Tracking shots of these isosurface plots are part of supplementary movies 3 and 4 .

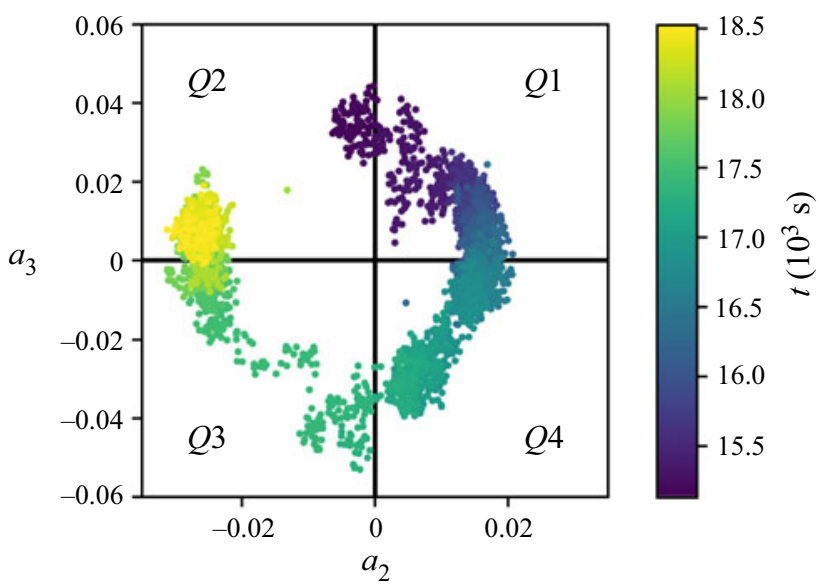

FIgURE 14. Phase space of the modes 2 and 3 during a reconfiguration event.

More information about the characteristics of the present reconfiguration event can be deduced from its progression in the phase space of the time coefficients of modes 2 and 3 displayed in figure 14. It shows, that approximately three quarters of the reconfiguration cycle are covered by the measurement, which is consistent with the temporal consideration of figure $9(a)$. The distribution of flow states in this phase plane further reveals an elliptical shape. While it is almost axisymmetric to $a_{3}=0$, its extent in the $a_{2}$ direction is larger in the negative direction. This might be caused by parts of the secondary roll branch inherent to this mode. Another possible explanation is that negative $a_{2}$ values represent the flow state which is preferred in comparison with the reversed one.

Besides that eccentricity, the point density reveals the rate at which the states change. This also indicates an accumulation and release process, as the state evolves slowly around $a_{3}=0$ as well as in quadrant $Q 4$ and rapidly in the quadrants $Q 1$ and $Q 3$.

Projecting the progression of $Q 4$ onto $Q 2$ yields point-symmetric change rates relative to the origin. This indicates that both diagonally aligned states of the main convection roll can be considered as meta-stable states with similar mechanisms depleting their stability. 

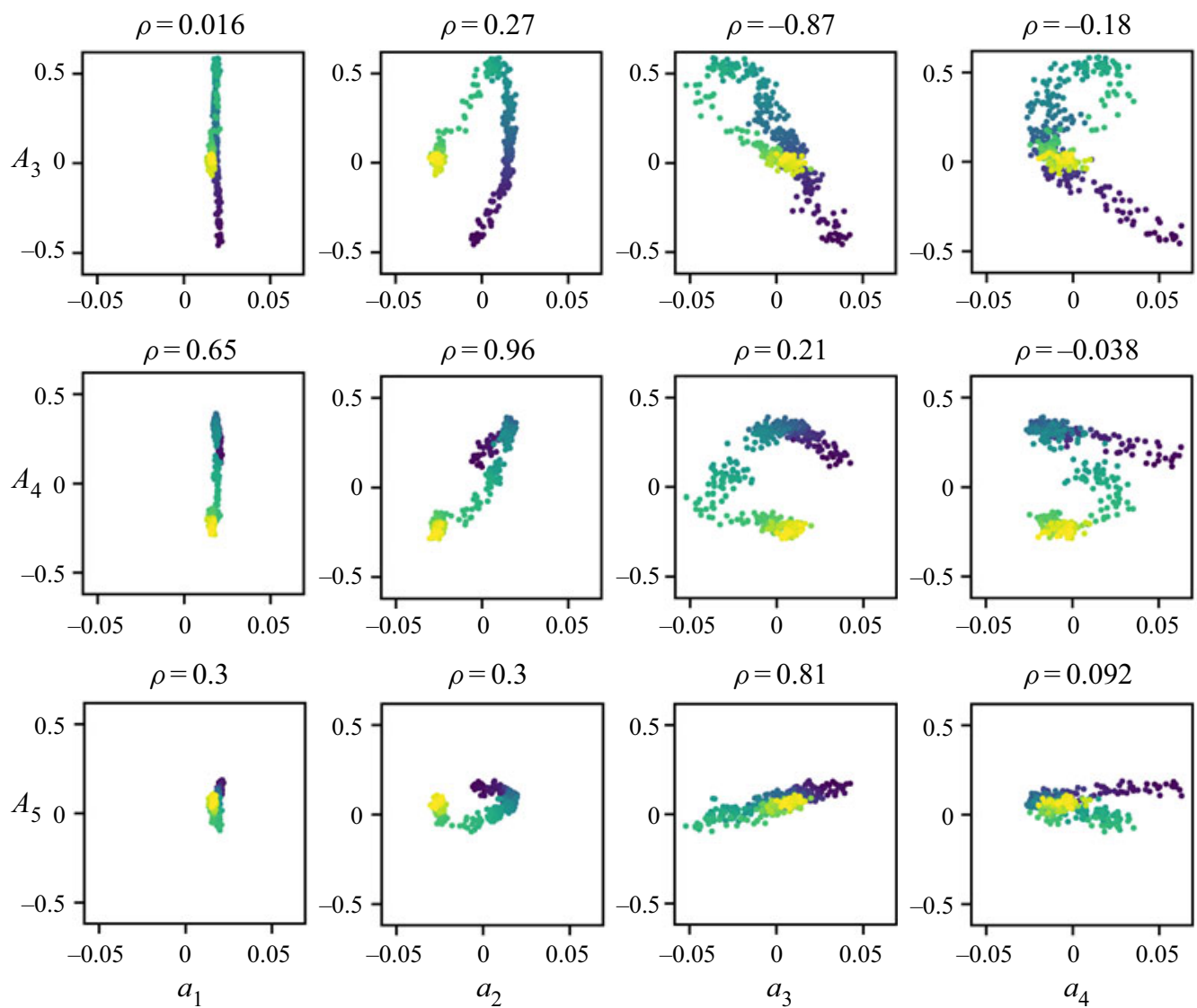

FIgURE 15. Correlation plots of the cosine coefficients $A$ and the POD coefficients $a$ with the respective Pearson's correlation coefficients $\rho$ for case $\mathcal{S}$.

To identify relations between the different dynamics of the temperature and velocity field, we conducted correlations of the cosine and POD coefficients, see figure 15 . It reveals that the prevalence of the 4 LSC state $\left(A_{4}\right)$ strongly correlates with the respective coherent structure determined by the POD $\rho_{A_{4} a_{2}}=0.96$. During the events, both $A_{3}, A_{5}$ and $a_{3}, a_{4}$ become particularly active. However, only $a_{3}$ yields reasonable correlation coefficients for $\rho_{A_{3} a_{3}}$ and $\rho_{A_{5} a_{3}}$ with differing signs. However, these correlations may be coincidental, as the structure of mode 3 itself does not contain flow structures explaining rear-wall hot spots, whose positions would match the implied wavenumbers. As the cosine functions cover the complete sample, connecting features between the temperatures and the flow process represented by mode 3 might also exist outside the PIV domain. Regarding $a_{4}$, only low correlation values are registered. However, the ' $<$ '-shape of the correlation plots indicates that there is a correlation with the absolute values of $\left|A_{3}\right|$ and $\left|A_{4}\right|$. That means this mode is associated with the transition period characterised by the hot spot movement. This also explains that, the mode's contribution is of the same sign for both directions of the reversal of mode 2 .

In summary, the POD provided coherent structures to generate a model concept for the reconfiguration events. It includes constant (mode 1) and reversing (mode 2) fractions of the flow structure. Modes 3 and 4 represent details of the two reversals processes of 
mode 2, which are dependent and independent from the reversal direction, respectively. In particular, the separation of the superimposed forced flow (mode 1) allowed us to consider these events as buoyancy-induced flow reversals with an accumulation and release process.

The correlation plots of the latter two modes showed that there is a correlation of these modes and the cosine fit amplitudes which describe the translation mechanism. Nevertheless, the causal connection cannot be deduced from the modes' coherent structures in an obvious manner. Therefore both concepts, translation and switching, represent projections of a complex underlying flow process. Thus, their connection should be further investigated in the future.

\subsection{Velocity fields of continuous reconfigurations}

As discussed in $\S 4.1$, the wavenumbers 7, 8 and 9 are dominant at certain times of a reconfiguration cycle for the smaller Richardson number $R i_{\mathcal{C}}=1.5$. In figure $16(a)$, we depict coefficient $A_{8}$, which showed the largest amplitudes, to emphasise how the acquired flow fields are associated with the cycle. The graph shows approximately six cycles of varying amplitudes of which just over four were covered by PIV. Six exemplary flow fields of that measurement are shown in figure $16(b)$ analogous to $\S 4.2$.

Snapshot I shows a convection roll which is straight in relation to the different alignments of case $\mathcal{S}$. However, with regard to the color-coded $Y$-positions, it is possible to observe a displacement towards the rear at the sides of the measurement volume and towards the front at $X \approx L / 8$. That equals a doubled number of distinct diagonal alignments in the PIV domain in comparison with case $\mathcal{S}$. It further matches the dominant wavenumber $w=8$ derived from the temperature data by the cosine fits. For snapshot II, there are no roll displacements from the centre. This coincides with a constant decline and zero crossing of $A_{8}$. The next snapshot (III) relates to a minimum of $A_{8}$ and shows the opposite displacements in the velocity field as compared to I. Snapshot IV completes the cycle since it again represents a zero crossing of $A_{8}$ with no roll displacement observable in the corresponding velocity field. To demonstrate the repeatability, the snapshots $\mathrm{V}$ and VI show similar roll core arrangements as I and III. Both pairs represent a maximum and a minimum of $A_{8}$, respectively.

Just as in case $\mathcal{S}$, this case also shows a switching of the diagonal roll alignment, whereas the analysis of the temperature data indicates a translation. However, this case is characterised by different associations of the hot and cold regions at the rear wall with the main roll's $Y$-displacement: by comparing the implied temperature distribution of $A_{8}$ with the velocity fields, we found that warmer regions are associated with areas where the convection roll's core is displaced towards the rear. This is the opposite behaviour compared to the $\mathcal{S}$ case.

To better understand this observation, it is necessary to gain insight into the coherent structures of this flow by applying a POD to the velocity fields. Above all, these roll core displacements are superimposed by erratic fluctuations which have nearly the same amplitude as the displacement in the exemplary snapshots, as supplementary movie 5 associated with figure 16(b) shows. This emphasises the need for a filtering analysis of the flow structures also represented by the POD. Following the same approach as in $\S 4.2$, the eigenvalue distribution as well as the normalised structure of the most prevalent modes is depicted in figure 17.

For this case, the first mode includes significantly more energy $(79 \%)$ than the first mode of case $\mathcal{S}(60 \%)$. Since a larger amount of energy is concentrated within this mode, less energy is allocated to the following modes compared to their equivalents of case $\mathcal{S}$. For instance, the contribution of the second mode is already smaller than $1 \%$. 
(a)

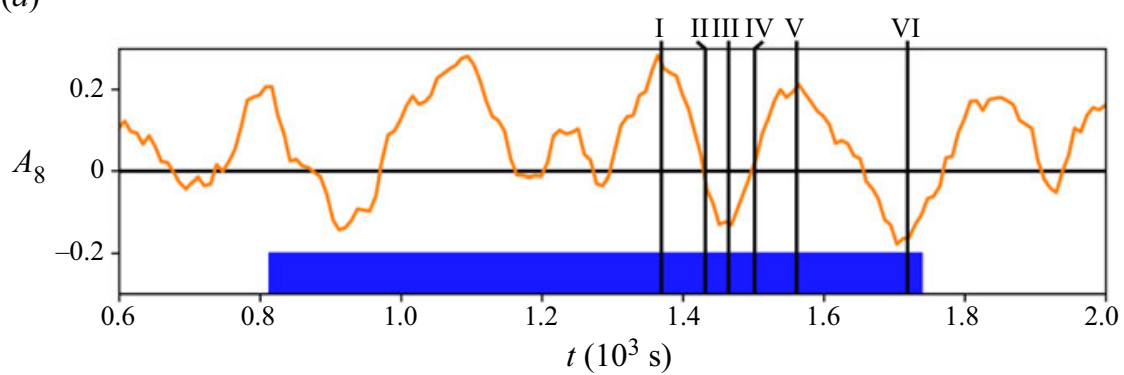

(b)
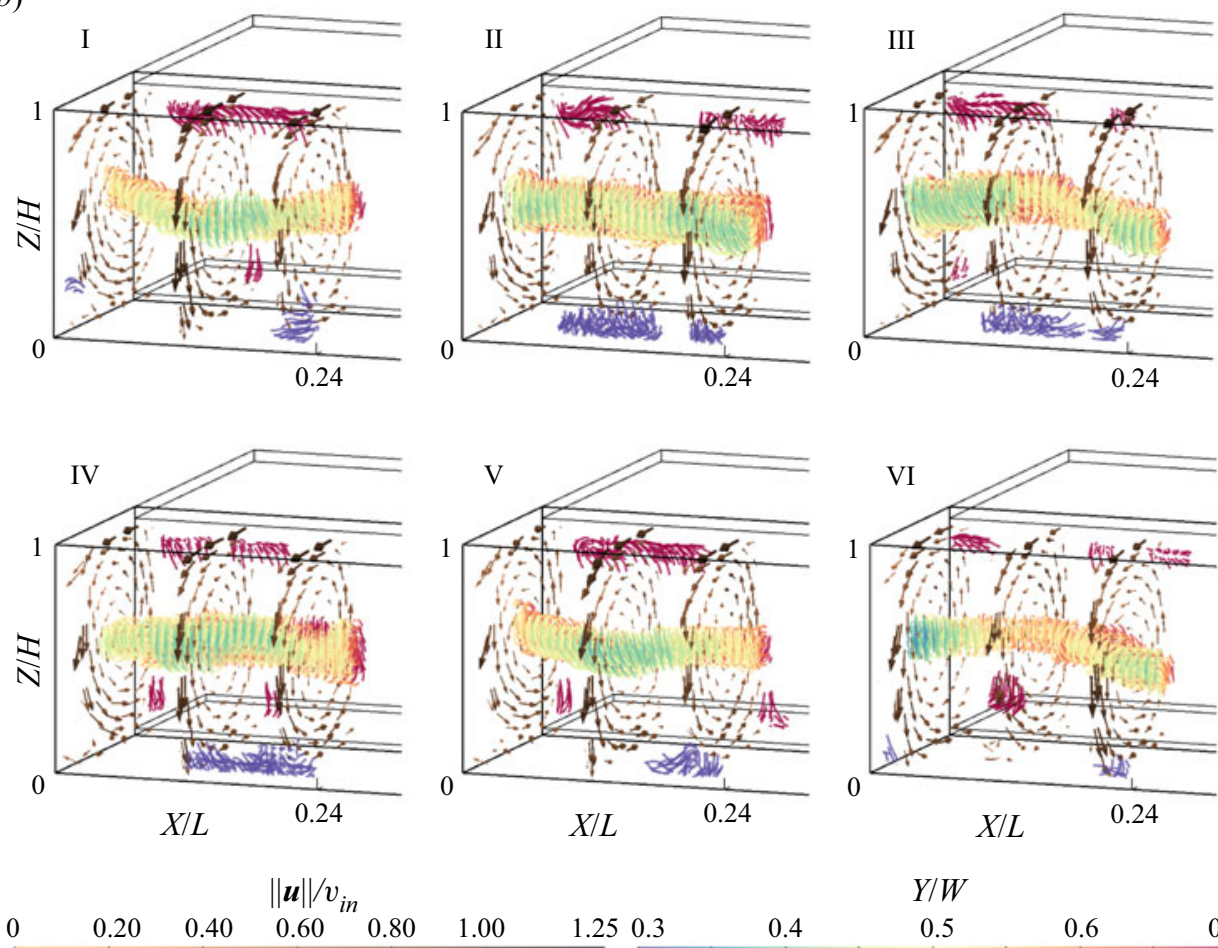

1.00

1.250 .3

0.4

$Y / W$

$0.5 \quad 0.6$ 0.7

FIGURE 16. (a) Time series of the wavenumber amplitudes over the course of a reconfiguration event. The interval for which velocity fields were measured is marked by a blue bar. (b) Velocity fields for 6 points in times, which are indicated in the temperature series. The fields are represented by vectors in 3 cross-sections and streamlines in areas of $\|\boldsymbol{u}\| / v_{\text {in }}<0.2$, which reflect the convection roll core. The displayed fields are short-time averaged over 25 frames. The complete time series can be seen in supplementary movie 5 .

This corresponds to the smaller Richardson number of case $\mathcal{C}$, as it signals that the inertial forces, which support the structure of the first mode, are even more dominant for this case.

Similar to case $\mathcal{S}$, the second mode incorporates coherent structures with rotation axes parallel to the $Y$-axis. In contrast to the single circulation which spreads across the whole domain for case $\mathcal{S}$, we observe six smaller circulations for the $\mathcal{C}$ case. Their arrangement, two rolls in the longitudinal direction and three of these pairs in the vertical direction of the domain, was similarly found by Westhoff (2012, pp. 57-59). 
(a)
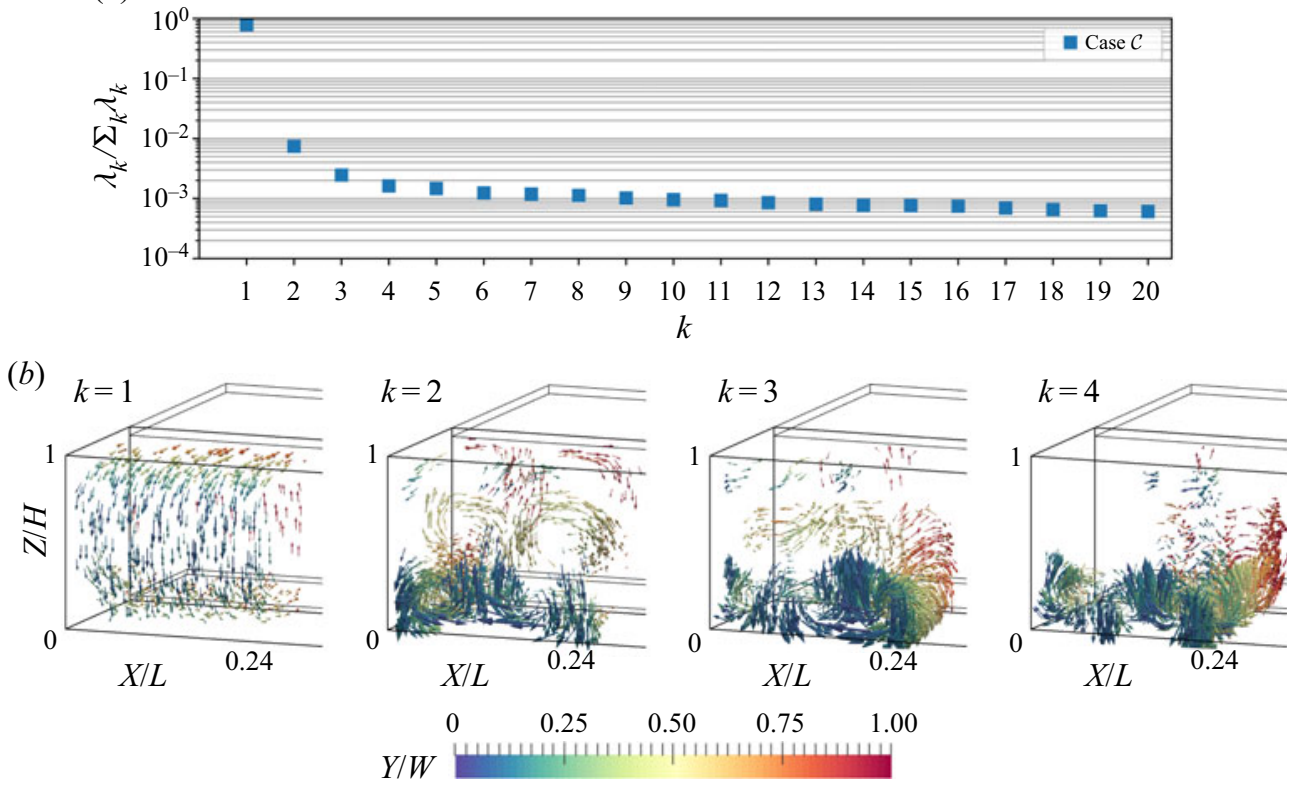

FIGURE 17. Eigenvalue distribution for the POD of case $\mathcal{C}(a)$ and the four most prevalent modes normalised by the square root of the respective eigenvalue $(b)$. Their structure is represented by the vectors of the highest magnitude. Supplementary movie 6 contains tracking shots of the displayed structures.

Modes 3 and 4 also display vortical structures, whose rotation axes are parallel to $Y$. However, their strongest contribution is limited to one layer of vortices above the bottom plate. Together with mode 2 and with regard to Brunton \& Kutz (2019, pp. 396-397), these structures represent the translation of vortices in this lower layer. This motion is not transferred to the centre of the sample, as contributions of modes 3 and 4 are weak in this region and a switching can be observed. Our analysis of these modes is limited to their structure, as the time development coefficients of modes with lower eigenvalues appear increasingly noisy.

Superimposing the first two modes yields, similar to case $\mathcal{S}$, diagonally aligned convection roll segments in the centre $X Y$-plane. Regarding mode 2, only the circulations at central $Z$-positions influence the alignment of the core of forced convection structure of mode 1, which is positioned at the same height. This explains the difference between both cases regarding the association of warm regions at the rear wall and the displacement of the convection roll core: while the centre pair of rotating coherent structures controls the roll displacement, the counter-rotating bottom structures affect the air reaching the temperature sensors.

In order to assess whether roll segments of this case perform a switching motion during reconfigurations, we consider the time development coefficients displayed in figure 18. Again, the first mode has a constant coefficient of just under 0.02, signalling a consistent contribution of the main forced convection roll structure to the flow. Whereas the second mode shows fluctuations around zero with varying amplitudes. The changes of sign of this mode's coefficients indicate that the direction of the coherent structures of mode 2 reverses for case $\mathcal{C}$, too. That means that the overall convection roll alignment switches as in case $\mathcal{S}$, but with the doubled spatial wavenumber. 


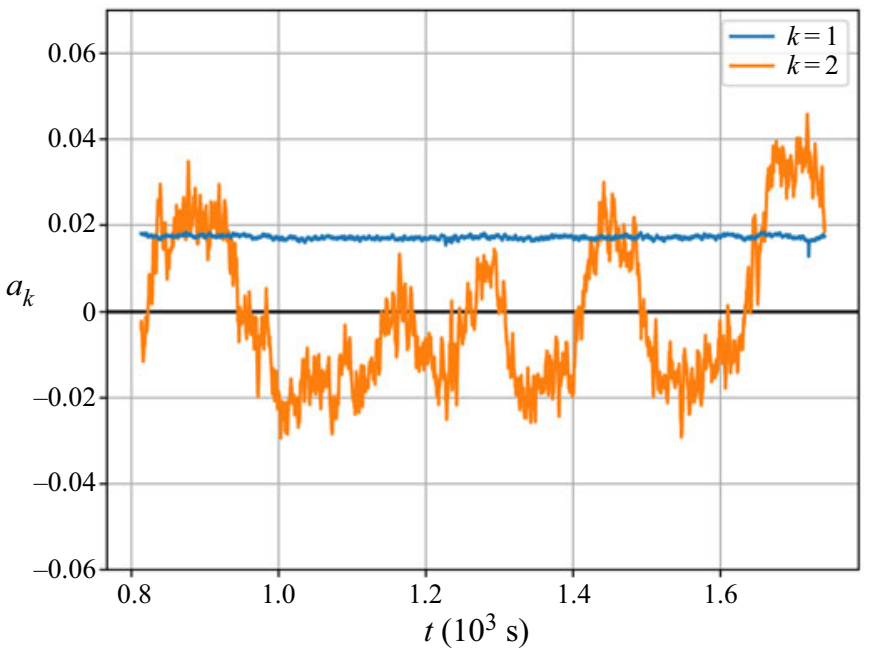

FIgURE 18. Time development coefficients for the first 2 POD modes also displayed in figure 17.

The relation between velocity and temperature fields is investigated by means of correlation plots presented in figure 19. The strongest correlation occurs with $\left|\rho_{A_{8} a_{2}}\right|=$ 0.83 which confirms that the lower $Y$-parallel roll pair of mode 2 is responsible for the appearance of LSC-indicating hot spots at the rear wall.

As stated in $\S 4.1$, the characteristics of the temperature fit parameters indicated a mechanism driven by forced flow. The second mode in case $\mathcal{C}$ confirms this concept: the strong bottom pair of coherent structures of this mode has a similar appearance as Taylor-Görtler-like vortices appearing in lid-driven or open cavity flows (Koseff \& Street 1984; Iwatsu et al. 1989). Both studies show that these structures can occur with a size of $H / 3$ for Re numbers of the same magnitude as those of the present study.

These structures originate from a Görtler or Taylor instability as the down-welling flow follows concave streamlines bending towards the bottom plate near the front edge of the sample. Both instabilities are based on an unstable radial stratification of the flow's angular momentum (Strutt 1917; Taylor 1923; Görtler 1954). Yet, the Taylor and Görtler numbers are tailored to determine the onset of unstable behaviour for the respective flows and are thus unsuitable to explain the different behaviour of the two present cases. Therefore, we apply the Rayleigh criterion for stability $(\Phi>0)$ presented by Chandrasekhar (2013, pp. 273-275). In order to compare both cases, the stability discriminant $\Phi=\left(1 / r^{3}\right) \nabla_{r}(r\|\boldsymbol{u}\|)^{2}$ is calculated for the flow fields averaged over complete respective time series. These fields are similar to the first modes and thereby contain the longitudinal roll structure from which Taylor-Görtler-like vortices eventually arise. All details regarding the definition of $\Phi$ can be found in appendix A.

Figure 20 shows the $\Phi$ fields in a representative cross-section at $X=L / 8$. For both cases, regions of unstable angular momentum stratifications $(\Phi<0)$ can be found next to the edge vortices in the top and bottom left corners of the cross-sections, where the vortex boundaries (see appendix A) are highlighted by green contour lines. The decisive difference between the cases is that the instability regions occurring for case $\mathcal{C}$ at the bottom edge vortex are stronger and larger. Integrating the regions of negative $\Phi$ next of the bottom edge vortex yields $-0.018 \mathrm{~m}^{2} \mathrm{~s}^{-2}$ for case $\mathcal{S}$ and $-0.056 \mathrm{~m}^{2} \mathrm{~s}^{-2}$ for case $\mathcal{C}$. 

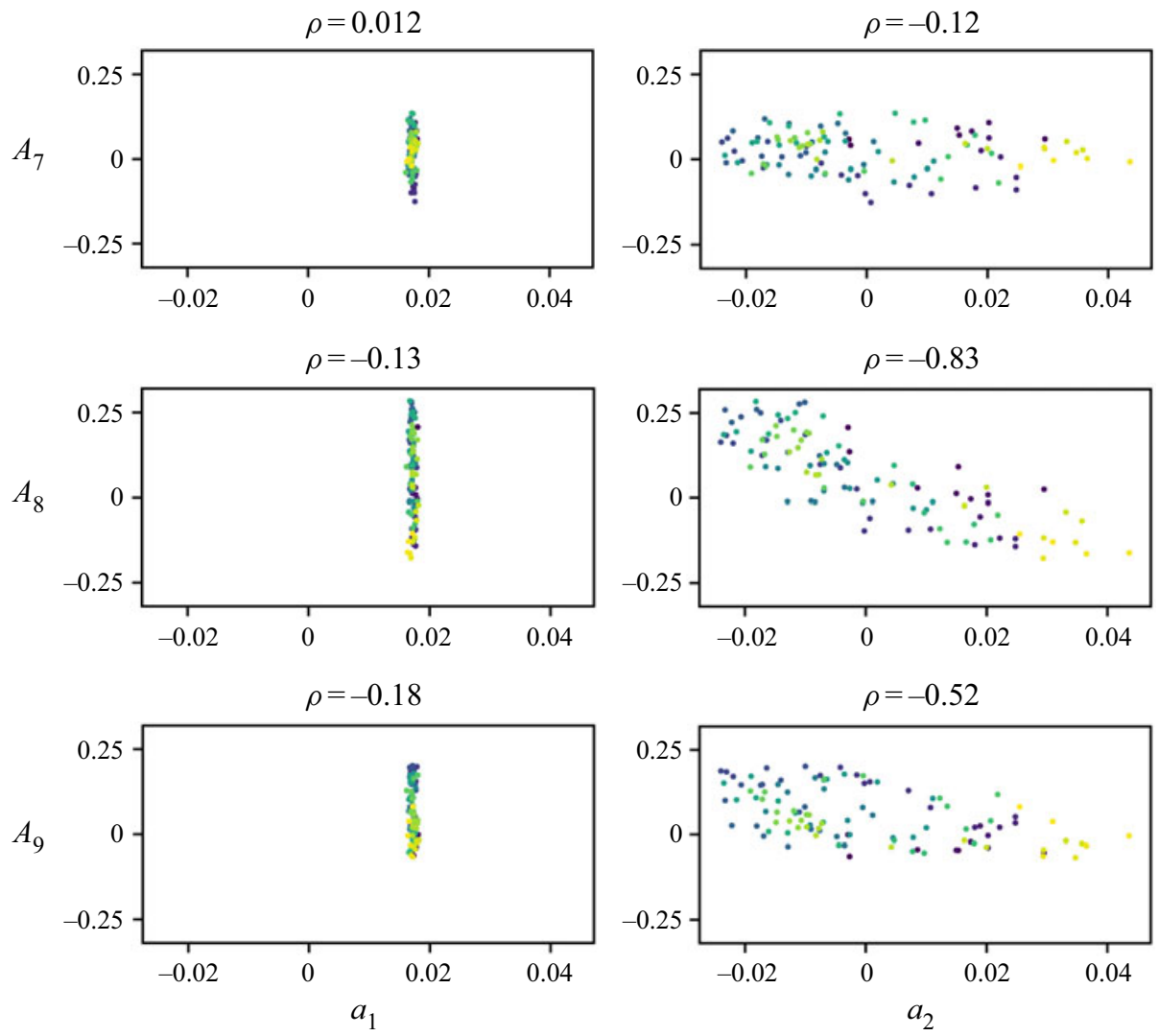

FIGURE 19. Correlation plots of the cosine coefficients $A$ and the POD coefficients $a$ with the respective Pearson's correlation coefficients $\rho$ for case $\mathcal{C}$.

This proves that the centrifugal forces, which can induce Taylor-Görtler-like vortices, are significantly stronger for the low $R i$.

To sum it up, the different behaviour of both cases can be attributed to the different manifestations of their longitudinal convection rolls. While they appear similar in figures 11 and 17, the different relation between thermal and forced convection causes the inlet jet to curve dissimilarly around the front edges of the sample and consequently to develop different angular momentum gradients.

Besides displaying similar vortex structures as case $\mathcal{C}$, open cavity flow also holds analogies for the observed dynamic behaviour of these structures. Douay, Pastur \& Lusseyran (2016) and Picella et al. (2018) presented cases of these flows where sidewall effects lead to an instability of the arrangement of Taylor-Görtler-like vortices. Such effects lead to Bödewadt flow (Schlichting \& Gersten 2016, pp. 329-330). However, this type of flow alone would induce a movement of the structures from the centre to the sidewalls of the sample. Hence, this mechanism can only function as an initial stimulus, while the switching process is then driven by an interaction of both buoyancy and inertial forces. Therefore, future research should focus on the proposed interaction, for example with parameter studies regarding the exact onset of this reconfiguration type. 

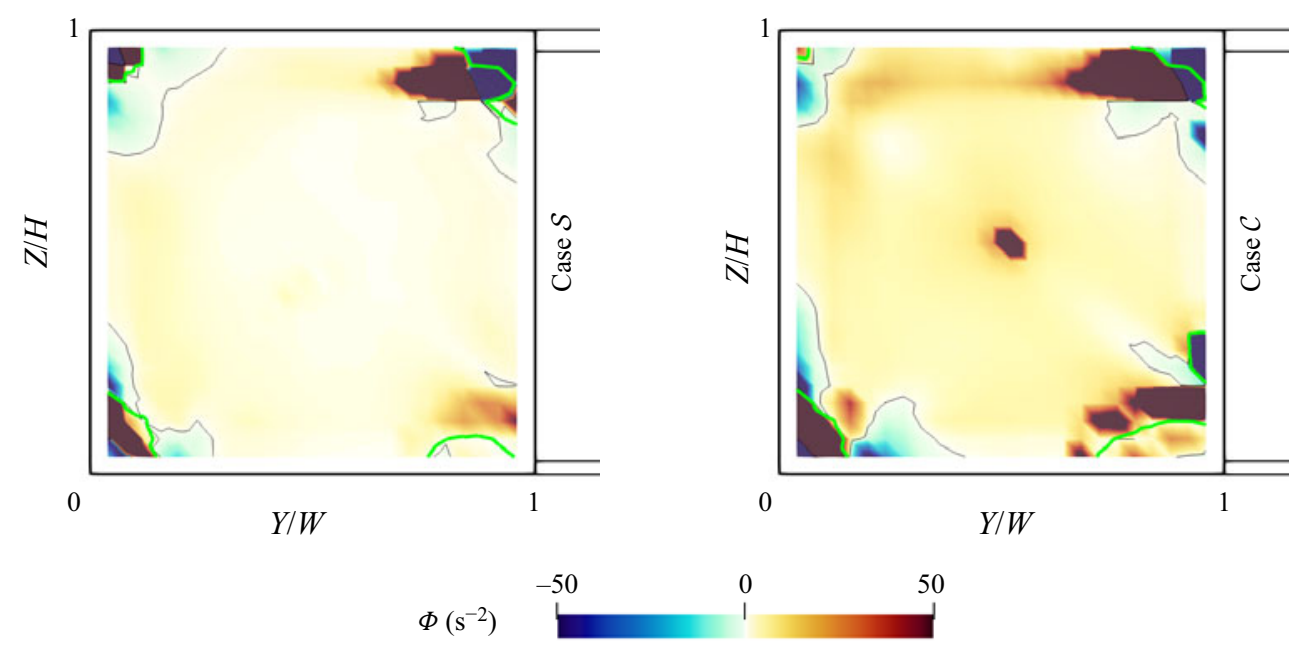

FIGURE 20. Value of $\Phi$ at $X=L / 8$ of the average velocity field for both cases. The thin black contour line highlights the change of sign. The thicker green contour indicates the extent of the edge vortices, as it shows where the $X$-curvature equals zero.

\section{Conclusion and outlook}

Mixed convection flow in a cuboidal sample was investigated for two cases of different Richardson numbers, $R i_{\mathcal{S}}=3.7$ and $R i_{\mathcal{C}}=1.5$, in order to determine to what extent concepts of RBC can be transferred to the occurring spontaneous or continuous flow reconfigurations. An initial rear-wall temperature analysis allowed us to allocate different prevalent numbers of LSCs, namely $w_{\mathcal{S}}=4$ and $w_{\mathcal{C}}=8$, to the cases. For both cases, the temperature measurements confirmed the translation of the structures observed by Schmeling et al. (2013). We showed that this translation can be described by fitting a sum of cosine functions to the temperature distribution. The time evolution of the fit parameters implied - in accordance with earlier findings - that the apparent translation is accompanied by dissolving and emerging LSCs near the sidewalls. However, 3-D PIV measurements of the volume next to the left sidewall were analysed for both cases and showed that the translation mechanism cannot be transferred to the dynamics of the velocity structures. Instead, a switching of the observable diagonal section(s) over the course of a reconfiguration was identified.

In accordance with $\mathrm{RBC}$ in rectangular samples, a secondary circulation in shape of a convection roll branch was found for the case of spontaneous reconfigurations $(\mathcal{S})$. However, it rather manifests a given LSC structure than it influences the reconfiguration process. The POD of the observed $\mathcal{S}$-type event extracted a set of influential modes. One mode represented a large fraction of the thermally forced flow as it resembles the flow structure of pure RBC. It allowed us to consider the switching of a diagonal LSC as superposition of constant forced flow combined with a reversal of the thermal flow components. Furthermore, the time evolution of the cosine and POD coefficients revealed that a process of accumulation and release is the underlying reconfiguration concept of this case. In particular, the events are associated with the release of multiple strong plumes from the front bottom edge vortex.

The POD results for the continuous reconfiguration case $(\mathcal{C})$ showed that the second mode also supports the concept of switching diagonals, which was observed in the 
velocity fields of this case, too. The second mode's structure of vertically stacked coherent circulations further explained the opposite relation between convection roll displacement and hot spots in comparison with case $\mathcal{S}$. The stacked structures additionally indicated that this case including its reconfigurations is mainly driven by forced convection. We found that these structures form due to the predominant influence of the Taylor or Görtler instability. A consideration of similar structures in open cavity flow shows that secondary instabilities provided also explanations for the onset of reconfigurations of this structure.

Correlations between the different time coefficients obtained from the temperature and velocity fields showed that the respective second mode covers the formation of the prevalent temperature structure for both cases. However, many aspects of the relation between the different flow structure dynamics, translation of temperatures and switching of velocities, are still not understood. Future research should focus on the coupling of both mechanisms and on how the different states affect the heat transport in the sample.

Overall this study showed that certain concepts of RBC are transferable to mixed convection. As case $\mathcal{S}$ showed, it is possible to approximate the reconfigurations as a reversal of the thermal fractions of the flow, while the forced fractions remain constant. However, this simplification is only valid for high enough $R i$. This was shown by the investigation of the low- $R i$ case $\mathcal{C}$, which had nearly the same $R a$ as case $\mathcal{S}$ but showed completely different coherent structures.

This raises the question of a critical $R i$ for this distinction, as it may not necessarily be bound to the reconfiguration type and is therefore a topic for future research. Future investigations should also focus on confirming the implications for the complete sample which are so far only extrapolations, due to the technical limitations of 3-D PIV. The present study provides an important prerequisite, as the knowing the three-dimensional structure of a complete LSC provides the basis for interpretation of future two-dimensional measurements covering the complete sample.

\section{Acknowledgements}

The authors thank A. Köhne for her support provided by proofreading the manuscripts.

\section{Declaration of interests}

The authors report no conflict of interest.

\section{Supplementary movies}

Supplementary movies are available at https://doi.org/10.1017/jfm.2020.705.

\section{Appendix A. Calculation of the Rayleigh criterion discriminant}

In $\S 4.3$, the reason for the existence of Taylor-Görtler-like vortices is explained by comparing the fields of the Rayleigh criterion discriminant $\Phi=\left(1 / r^{3}\right) \nabla_{r}(r\|\boldsymbol{u}\|)^{2}$. This definition is a modification of the definition in cylindrical coordinates by Chandrasekhar (2013, pp. 273-275)

$$
\Phi=\frac{1}{R^{3}} \frac{\mathrm{d}}{\mathrm{d} R}\left(R^{2} \Omega\right)^{2} .
$$

The origin of this cylindrical coordinate system with the radius coordinate $R$ is assumed to be the centre of an analysed vortex. As the position of this centre is not per se known, we replaced $R$ by the local curvature radius $r$ and its associated unity vector $r$. The latter is 
used for the directional derivative of the squared angular momentum term $\left(R^{2} \Omega\right)^{2}$, where the azimuthal velocity $\Omega=\|\boldsymbol{u}\| / r$ is replaced by the velocity magnitude $\|\boldsymbol{u}\|$, as each velocity vector is tangential to its local rotation.

In order to calculate the local curvature radii, we refer to Theisel (1996, pp. 22-23). As a first step, the curvature vector $\boldsymbol{\kappa}$ is calculated as follows:

$$
\kappa=\frac{u \times \dot{u}}{\|u\|^{3}} .
$$

This introduces the time derivative $\dot{\boldsymbol{u}}$ of the velocity vector. As we analyse single vector fields as steady states, it is reduced to the convection term

$$
\dot{u}=(u \cdot \nabla) \cdot u .
$$

The local radius $r$ and its unity vector $\boldsymbol{r}$ are then calculated as follows:

$$
\begin{gathered}
r=\frac{1}{\|\boldsymbol{\kappa}\|}, \\
r=\frac{\boldsymbol{u} \times \boldsymbol{\kappa}}{\|\boldsymbol{u} \times \boldsymbol{\kappa}\|} .
\end{gathered}
$$

For figure 20, these equations were applied in paraview, which calculates derivatives for the cells inscribed by the grid points of the vector field. These cell values were then interpolated to the original grid to complete the calculations. The curvature vector was further used to highlight the boundaries of the edge vortices, which we defined as $\kappa_{X}=0$.

\section{REFERENCES}

Araujo, F. F., Grossmann, S. \& Lohse, D. 2005 Wind reversals in turbulent Rayleigh-Bénard convection. Phys. Rev. Lett. 95, 084502.

BAI, K., Ji, D. \& BROWn, E. 2016 Ability of a low-dimensional model to predict geometry-dependent dynamics of large-scale coherent structures in turbulence. Phys. Rev. E 93 (2), 023117.

Blass, A., Zhu, X., Verzicco, R., Lohse, D. \& Stevens, R. J. A. M. 2020 Flow organization and heat transfer in turbulent wall sheared thermal convection. J. Fluid Mech. 897, A22.

Brown, E. \& Ahlers, G. 2006 Rotations and cessations of the large-scale circulation in turbulent Rayleigh-Bénard convection. J. Fluid Mech. 568, 351.

Brown, E. \& Ahlers, G. 2009 The origin of oscillations of the large-scale circulation of turbulent Rayleigh-Bénard convection. J. Fluid Mech. 638, 383-400.

Brunton, S. L. \& KutZ, J. N. 2019 Data-Driven Science and Engineering: Machine Learning, Dynamical Systems, and Control. Cambridge University Press.

Castillo-Castellanos, A., Sergent, A., Podvin, B. \& Rossi, M. 2019 Cessation and reversals of large-scale structures in square Rayleigh-Bénard cells. J. Fluid Mech. 877, 922-954.

Castillo-Castellanos, A., Sergent, A. \& Rossi, M. 2016 Reversal cycle in square Rayleigh-Bénard cells in turbulent regime. J. Fluid Mech. 808, 614-640.

Chandrasekhar, S. 2013 Hydrodynamic and Hydromagnetic Stability. Dover Publications.

Chen, X., Huang, S.-D., XiA, K.-Q. \& XI, H.-D. 2019 Emergence of substructures inside the large-scale circulation induces transition in flow reversals in turbulent thermal convection. J. Fluid Mech. 877, R1.

Chen, X., WANG, D.-P. \& XI, H.-D. 2020 Reduced flow reversals in turbulent convection in the absence of corner vortices. J. Fluid Mech. 891, R5.

Cioni, S., Ciliberto, S. \& Sommeria, J. 1997 Strongly turbulent Rayleigh-Bénard convection in mercury: comparison with results at moderate Prandtl number. J. Fluid Mech. 335, 111-140. 
Clever, R. M. \& Busse, F. H. 1991 Instabilities of longitudinal rolls in the presence of Poiseuille flow. J. Fluid Mech. 229, 517-529.

Douay, C. L., Pastur, L. R. \& Lusseyran, F. 2016 Centrifugal instabilities in an experimental open cavity flow. J. Fluid Mech. 788, 670-694.

Foroozani, N., Niemela, J. J., Armenio, V. \& Sreenivasan, K. R. 2017 Reorientations of the large-scale flow in turbulent convection in a cube. Phys. Rev. E 95, 033107.

Funfschilling, D. \& Ahlers, G. 2004 Plume motion and large-scale circulation in a cylindrical Rayleigh-Bénard cell. Phys. Rev. Lett. 92 (19), 194502.

FunfsChILling, D., BROWN, E. \& AHLERs, G. 2008 Torsional oscillations of the large-scale circulation in turbulent Rayleigh-Bénard convection. J. Fluid Mech. 607, 119-139.

GÖRTLER, H. 1954 On the three-dimensional instability of laminar boundary layers on concave walls. Technical Memorandum. National Advisory Committee for Aeronautics.

Huang, S.-D., WANG, F., XI, H.-D. \& XIA, K.-Q. 2015 Comparative experimental study of fixed temperature and fixed heat flux boundary conditions in turbulent thermal convection. Phys. Rev. Lett. 115 (15), 154502.

HUANG, S.-D. \& XIA, K.-Q. 2016 Effects of geometric confinement in quasi-2-d turbulent Rayleigh-Bénard convection. J. Fluid Mech. 794, 639-654.

IEC 2008 Industrial platinum resistance thermometers and platinum temperature sensors. International Standard. International Electrotechnical Commission.

Iwatsu, R., Ishit, K., Kawamura, T., Kuwahara, K. \& HyUn, J. M. 1989 Numerical simulation of three-dimensional flow structure in a driven cavity. Fluid Dyn. Res. 5 (3), 173-189.

Kaczorowski, M. \& Wagner, C. 2009 Analysis of the thermal plumes in turbulent Rayleigh-Bénard convection based on well-resolved numerical simulations. J. Fluid Mech. 618, 89-112.

KosefF, J. R. \& STREeT, R. L. 1984 The lid-driven cavity flow: a synthesis of qualitative and quantitative observations. J. Fluids Engng 106 (4), 390-398.

KÜHN, M., EHRENFrIED, K., BosbaCH, J. \& WAGNER, C. 2011 Large-scale tomographic particle image velocimetry using helium-filled soap bubbles. Exp. Fluids 50 (4), 929-948.

KÜHN, M., EHRENFRIED, K., BosbACH, J. \& WAGNER, C. 2012 Large-scale tomographic PIV in forced and mixed convection using a parallel SMART version. Exp. Fluids 53 (1), 91-103.

Mishra, D., Muralidhar, K. \& MUnshi, P. 1999 A robust MART algorithm for tomografic applications. Numer. Heat Transfer B 35 (4), 485-506.

Mommert, M., Schiepel, D., Schmeling, D. \& Wagner, C. 2019 A flow-intrinsic trigger for capturing reconfigurations in buoyancy-driven flows in automated PIV. Meas. Sci. Technol. 30 (4), 045301.

Ni, R., HuAnG, S.-D. \& XiA, K.-Q. 2015 Reversals of the large-scale circulation in quasi-2D Rayleigh-Bénard convection. J. Fluid Mech. 778, R5.

Niehaus, K. A., Mommert, M., Schiepel, D., Schmeling, D. \& Wagner, C. 2020 Comparison of two unstable flow states in turbulent mixed convection. In New Results in Numerical and Experimental Fluid Mechanics XII (ed. A. Dillmann, G. Heller, E. Krämer, C. Wagner, C. Tropea \& S. Jakirlić), pp. 543-552. Springer International Publishing.

Niemela, J. J., Skrbek, L., Sreenivasan, K. R. \& Donnelly, R. J. 2001 The wind in confined thermal convection. J. Fluid Mech. 449, 169-178.

Pabiou, H., Mergui, S. \& BÉnARd, C. 2005 Wavy secondary instability of longitudinal rolls in Rayleigh-Bénard-Poiseuille flows. J. Fluid Mech. 542, 175-194.

Picella, F., Loiseau, J.-C., Lusseyran, F., Robinet, J.-C., Cherubini, S. \& Pastur, L. 2018 Successive bifurcations in a fully three-dimensional open cavity flow. J. Fluid Mech. 844, 855-877.

Pirozzoli, S., Bernardini, M., Verzicco, R. \& Orlandi, P. 2017 Mixed convection in turbulent channels with unstable stratification. J. Fluid Mech. 821, 482-516.

Podvin, B. \& SERGENT, A. 2012 Proper orthogonal decomposition investigation of turbulent Rayleigh-Bénard convection in a rectangular cavity. Phys. Fluids 24 (10), 105106.

Podvin, B. \& SERGENT, A. 2015 A large-scale investigation of wind reversal in a square Rayleigh-Bénard cell. J. Fluid Mech. 766, 172-201. 
Podvin, B. \& Sergent, A. 2017 Precursor for wind reversal in a square Rayleigh-Bénard cell. Phys. Rev. E 95 (1), 013112.

QIU, X.-L., Shang, X.-D., Tong, P. \& XIA, K.-Q. 2004 Velocity oscillations in turbulent Rayleigh-Bénard convection. Phys. Fluids 16 (2), 412-423.

Resagk, C., Du Puits, R., Thess, A., Dolzhansky, F. V., Grossmann, S., Fontenelearaujo, F. \& LOHSE, D. 2006 Oscillations of the large scale wind in turbulent thermal convection. Phys. Fluids 18 (9), 095105.

Schlichting, H. \& Gersten, K. 2016 Boundary-Layer Theory. Springer.

SCHMELING, D., BosBACH, J. \& WAGNER, C. 2013 Oscillations of the large-scale circulation in turbulent mixed convection in a closed rectangular cavity. Exp. Fluids 54 (5), 1517.

Schmeling, D., Westhoff, A., Kühn, M., Bosbach, J. \& Wagner, C. 2011 Large-scale flow structures and heat transport of turbulent forced and mixed convection in a closed rectangular cavity. Intl J. Heat Fluid Flow 32 (5), 889-900.

SiRovich, L. 1987 Turbulence and the dynamics of coherent structures. I - Coherent structures. II Symmetries and transformations. III - Dynamics and scaling. Q. Appl. Maths 45, 561-571.

Soucasse, L., Podvin, B., Rivière, P. \& Soufiani, A. 2019 Proper orthogonal decomposition analysis and modelling of large-scale flow reorientations in a cubic Rayleigh-Bénard cell. J. Fluid Mech. 881, 23-50.

Sreenivasan, K. R., Bershadskit, A. \& Niemela, J. J. 2002 Mean wind and its reversal in thermal convection. Phys. Rev. E 65 (5), 056306.

Strutt, J. W. 1917 On the dynamics of revolving fluids. Proc. R. Soc. Lond. A 93 (648), 148-154.

Sugiyama, K., Ni, R., Stevens, R. J. A. M., Chan, T. S., Zhou, S.-Q., Xi, H.-D., Sun, C., Grossmann, S., XiA, K.-Q. \& LOHSE, D. 2010 Flow reversals in thermally driven turbulence. Phys. Rev. Lett. 105, 034503.

Sun, C., XIA, K.-Q. \& Tong, P. 2005 Three-dimensional flow structures and dynamics of turbulent thermal convection in a cylindrical cell. Phys. Rev. E 72, 026302.

TAYloR, G. I. 1923 VIII. Stability of a viscous liquid contained between two rotating cylinders. Phil. Trans. R. Soc. Lond. A 223 (605-615), 289-343.

TheISEL, H. 1996 Vector field curvature and applications. PhD thesis, Universität Rostock.

Vasiliev, A., Frick, P., Kumar, A., Stepanov, R., Sukhanovskit, A. \& Verma, M. K. 2019 Transient flows and reorientations of large-scale convection in a cubic cell. Intl Commun. Heat Mass Transfer 108, 104319.

VILlermauX, E. 1995 Memory-induced low frequency oscillations in closed convection boxes. Phys. Rev. Lett. 75, 4618-4621.

WANG, Y., LAI, P.-Y., SONG, H. \& TONG, P. 2018 Mechanism of large-scale flow reversals in turbulent thermal convection. Sci. Adv. 4 (11), eaat7480.

Wessels, M., Schmeling, D., Bosbach, J. \& Wagner, C. 2019 On the impact of the aspect ratio on the formation of large-scale structures in turbulent mixed convection in a cuboidal sample. Intl $J$. Heat Fluid Flow 76, 231-241.

Westerweel, J. \& Scarano, F. 2005 Universal outlier detection for PIV data. Exp. Fluids 39 (6), $1096-1100$.

Westhoff, A. 2012 Spatial scaling of large-scale circulations and heat transport in turbulent mixed convection. PhD thesis, Georg-August-Universität Göttingen.

Westhoff, A., Bosbach, J., Schmeling, D. \& Wagner, C. 2010 Experimental study of low-frequency oscillations and large-scale circulations in turbulent mixed convection. Intl J. Heat Fluid Flow 31 (5), 794-804.

WiENEKE, B. 2008 Volume self-calibration for 3D particle image velocimetry. Exp. Fluids 45 (4), $549-556$.

XI, H.-D. \& XIA, K.-Q. 2007 Cessations and reversals of the large-scale circulation in turbulent thermal convection. Phys. Rev. E 75, 066307.

XI, H.-D., Zhou, S.-Q., Zhou, Q., Chan, T.-S. \& XIA, K.-Q. 2009 Origin of the temperature oscillation in turbulent thermal convection. Phys. Rev. Lett. 102, 044503.

XIA, K.-Q. 2013 Current trends and future directions in turbulent thermal convection. Theor. Appl. Mech. Lett. 3 (5), 052001. 
XIE, Y.-C., Ding, G.-Y. \& XIA, K.-Q. 2018 Flow topology transition via global bifurcation in thermally driven turbulence. Phys. Rev. Lett. 120, 214501.

XIE, Y.-C., WEI, P. \& XIA, K.-Q. 2013 Dynamics of the large-scale circulation in high-Prandtl-number turbulent thermal convection. J. Fluid Mech. 717, 322-346.

Zhang, S., XiA, Z., Zhou, Q. \& Chen, S. 2020 Controlling flow reversal in two-dimensional Rayleigh-Bénard convection. J. Fluid Mech. 891, R4. 